\title{
Singular Vectors and Topological Theories FROM VIRASORO CONSTRAINTS VIA THE Kontsevich-Miwa TRANSFORM
}

\author{
B. Gato-Rivera \\ Instituto de Matemáticas y Física Fundamental, Serrano 123, Madrid 28006, Spain \\ and
}

A. M. Semikhatov

CERN, CH-1211 Geneva 23, Switzerland

and

P. N. Lebedev Physics Institute

Leninsky pr. 53, Moscow 117924, Russia

\begin{abstract}
We use the Kontsevich-Miwa transform to relate the different pictures describing matter coupled to topological gravity in two dimensions: topological theories, Virasoro constraints on integrable hierarchies, and a DDK-type formalism. With the help of the Kontsevich-Miwa transform, we solve the Virasoro constraints on the KP hierarchy in terms of minimal models dressed with a (free) Liouville-like scalar. The dressing prescription originates in a topological (twisted $N=2$ ) theory. The Virasoro constraints are thus related to essentially the $N=2$ null state decoupling equations. The $N=2$ generators are constructed out of matter, the 'Liouville' scalar, and $c=-2$ ghosts. By a 'dual' construction involving the reparametrization $c=-26$ ghosts, the DDK dressing prescription is reproduced from the $N=2$ symmetry. As a by-product we thus observe that there are two ways to dress arbitrary $d \leq 1 \bigcup d \geq 25$ matter theory, which allow its embedding into a topological theory. By the Kontsevich-Miwa transform, which introduces an infinite set of 'time' variables $t_{r}$, the equations ensuring the vanishing of correlators that involve BRST-exact primary states, factorize through the Virasoro generators expressed in terms of the $t_{r}$. The background charge of these Virasoro generators is determined in terms of the topological central charge $\mathrm{c} \neq 3$ as $\mathrm{Q}=\sqrt{\frac{3-\mathrm{c}}{3}}-2 \sqrt{\frac{3}{3-\mathrm{c}}}$.
\end{abstract}

CERN-TH.6752/92

IMAFF- $92 / 8$

hep-th@xxx/9212113

December 1992 


\section{Contents}

\begin{tabular}{lll}
\hline 1 & Introduction and discussion & 1
\end{tabular}

1.1 Virasoro constraints as decoupling equations . . . . . . . . . . . . . . . . . . . . 1

1.2 Solving Virasoro constraints by a 'target-space' theory . . . . . . . . . . . . . . 4

1.3 Decoupling equations as BRST invariance . . . . . . . . . . . . . . . . . . . 5

2 Constrained KP hierarchy and

$\begin{array}{ll}\text { the Kontsevich-Miwa transform } & 9\end{array}$

2.1 KP hierarchy . . . . . . . . . . . . . . . . . . . . . . . . . . . . . . . 9

2.2 Virasoro constraints . . . . . . . . . . . . . . . . . . . . . . . . . 10

2.3 Why the Kontsevich-Miwa transform? . . . . . . . . . . . . . . . . . . . . . . . 11

3 From the twisted $N=2$ and BRST invariance

$\begin{array}{ll}\text { to dressed null states } & 14\end{array}$

3.1 A 'mirror' BRST construction for the topological algebra . . . . . . . . . . . . . 14

3.2 Decoupling equations from BRST invariance . . . . . . . . . . . . . . . . . . 17

3.2 .1 Level-2 reduction and the decoupling equation . . . . . . . . . . . . . . . 18

3.2 .2 Level-3 reduction and the decoupling equation . . . . . . . . . . . . . . . 22

3.A Appendix: DDK formalism from $N=2 \ldots \ldots \ldots$. . . . . . . . . . . . . . . . . . 25

4 Dressed null states via a direct construction 29

4.1 Dressing at level 3 . . . . . . . . . . . . . . . . . . . 30

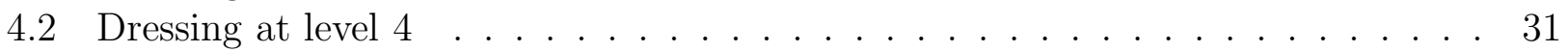

4.2 .1 Dressed null vectors over the '41' state . . . . . . . . . . . . . . . . . . . 31

4.2 .2 Dressed null vectors over the '22' state . . . . . . . . . . . . . . . . . . . 34

5 From dressed null states to the Virasoro constraints 38

5.1 Level-2 decoupling equation as Virasoro constraints . . . . . . . . . . . . . . . . 39

5.2 Virasoro constraints from level 3 . . . . . . . . . . . . . . . . . . . . . 40

5.2 .1 A naïve approach to level 3 . . . . . . . . . . . . . . . . . . . . . . . . . . . . . . . . . . 41

5.2 .2 Level-3 factorization once more . . . . . . . . . . . . . . . . . . . . . . . 44

5.3 Factorization at level 4 . . . . . . . . . . . . . . . . . . . . . . . . . . . 46

5.3 .1 Virasoro constraints from the '41' decoupling operator . . . . . . . . . . 46

5.3 .2 The ' 22 ' decoupling operator . . . . . . . . . . . . . . . . . . . 53

\begin{tabular}{|lll}
\hline 6 & Summary and generalizations & 54
\end{tabular}

\begin{tabular}{llr}
\hline 7 Concluding remarks & 58
\end{tabular}

\begin{tabular}{lr}
\hline References & 61
\end{tabular} 


\section{Introduction and discussion}

\subsection{Virasoro constraints as decoupling equations}

An important result of matrix models [1, 2, 3] was the discovery of an 'integrable' counterpart of the topological gravity + matter theories in two dimensions, in the form of constrained

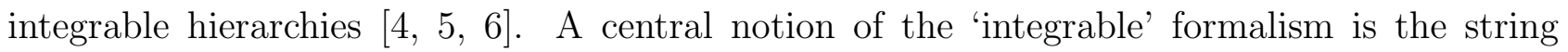

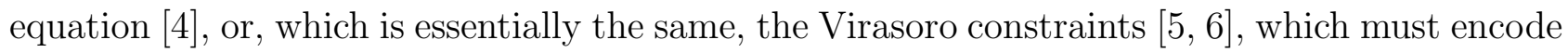
the 'dynamical' content of the theory and therefore must somehow be related to continuum field-theoretic formalism.

However, an immediate problem that one encounters when attempting to construct a direct correspondence between the integrable and the continuum formulations, is how to interpret in 'intrinsic' CFT terms the infinite collection of time parameters which label the integrable evolutions (in the language of topological theories the times appear as 'external' parameters that allow one to write down the generating function for the amplitudes [7, [1). In terms of these times $t_{r \geq 1}$, the most general Virasoro constraints on the tau function of the KP hierarchy read $\mathrm{L}_{p} \tau=0, p \geq-1$, where

$$
\begin{aligned}
\mathrm{L}_{p>0} & =\frac{1}{2} \sum_{s=1}^{p-1} \frac{\partial^{2}}{\partial t_{p-s} \partial t_{s}}+\sum_{s \geq 1} s t_{s} \frac{\partial}{\partial t_{p+s}}+\left(\mathrm{J}-\frac{1}{2}\right)(p+1) \frac{\partial}{\partial t_{p}} \\
\mathrm{~L}_{0} & =\sum_{s \geq 1} s t_{s} \frac{\partial}{\partial t_{s}} \\
\mathrm{~L}_{-1} & =\sum_{s \geq 1}(s+1) t_{s+1} \frac{\partial}{\partial t_{s}}
\end{aligned}
$$

with J being a free parameter円.

We will show that a conformal field theory can be recovered as a solution to these Virasoro constraints. To start with, recall that there does exist a transformation, known in the theory of integrable systems as the Miwa transform [14, 15], which expresses the (complexified) KP times through 'complex coordinates' living on the spectral parameter worldsheet:

\footnotetext{
${ }^{1}$ For $\mathrm{J}$ arbitrary, the constraints are more general than those actually derived from matrix models. However, our point of view is to promote to a 'first principle' the fact that appropriately constrained integrable hierarchies provide an alternative description of topological quantum gravity [9, 10, 11], which would make it natural to consider on an equal footing various constraints, including those whose derivation from a specific matrix model is not known (yet). This point of view has also been advocated previously in [12, 13].
} 


$$
t_{r}=\frac{1}{r} \sum_{j} n_{j} z_{j}^{-r}, \quad r \geq 1
$$

where $\left\{z_{j}\right\}$ is a set (infinite in order that the $t_{r}$ be independent) of points on the complex plane. As far as the integrable systems were concerned, the main interest in applications of (1.2) was concentrating around rewriting the integrable equations (taken in the Hirota form [16) as finite-difference equations in the $n_{j}$. On the other hand, in a context much closer to that of the present paper, Kontsevich [7] has used a parametrization for the time parameters similar to (1.2), in which, however, the $z_{j}$, rather than the $n_{j}$, were playing the 'active' role, while all the $n_{j}$ were set to a constant (see also [17]-[20]).

We will combine these two points of view on the transformation (1.2) by regarding it as a set of transformations from the complex coordinates $z_{j}$ to the times $t_{r}$, parametrized by the $n_{j}$, and call it the Kontsevich-Miwa transform [21]. The question now is how meaningful CFT data can be recovered by applying (1.2) to the KP hierarchy constrained by the operators (1.1).

A mathematical statement which we observe to underlie the sought correspondence, is formulated in Sect. 7 as a theorem on a class of differential operators. However, we would prefer to adopt a more physical standpoint. We are going to interpret the $z_{j}$ introduced via eq. (1.2) as positions of certain operator insertions in a conformal theory [15, 21]. The parameters $n_{j}$ will then provide a necessary freedom allowing us to relate the Virasoro constraints (1.1) to decoupling equations corresponding to null states in the conformal theory. The relation with the decoupling equations is a crucial step [21] that will allow us to go beyond the standard 'fermionic'/Grassmannian construction [16] for the tau function']. The decoupling equations are a primary tool in the analysis of $d<1$ models, and the study of the corresponding null vectors has a history of its own [23]-27]; the relevant structures are known to possess in certain cases relations to other problems in physics and mathematics [28, 29, 30. It will be amusing to find that the null vectors incorporate the set of $p \geq-1$ Virasoro constraints (obviously, the Virasoro generators $\mathrm{L}_{p}$ are quite distinct from the Virasoro generators represented on a CFT).

More specifically, the Kontsevich-Miwa transform relates the Virasoro constraints to minimal models that turn out to be dressed with an extra scalar that plays a similar rôle to that of the Liouville field in the formalism of [31, 32]. The Miwa parameters $n_{j}$ then acquire the meaning of the corresponding $U(1)$ 'Liouville' charges. The correspondence is achieved via an

\footnotetext{
${ }^{2}$ See also ref. [22] in which solutions to string equations were constructed in the Grassmannian language.
} 
ansatz 21] presenting the (Virasoro-constrained!) tau function as a correlator of a product of certain dressed primary field operators in a minimal model: For any value of the index $i$ chosen, the constraint

$$
\sum_{p \geq-1} z_{i}^{-p-2} \mathrm{~L}_{p} \tau=0
$$

determines the dependence of $\tau(t) \equiv \tau\{z\}$ on $z_{i}$ via

$$
\tau=\left\langle\ldots \Psi\left(z_{i}\right) \ldots\right\rangle
$$

where $\Psi$ is a dressed $(l, 1)$ or $(1, l)$ primary state (thus possessing a null descendant at level $l$ ), and the dots denote insertions at the other points $z_{j \neq i}$ of any of the $\left(l^{\prime}, 1\right)$ or $\left(1, l^{\prime}\right)$ dressed primary fields. Let us concentrate on just the one at $z_{i}$. The respective Miwa parameter $n_{i}$, which becomes the 'Liouville' $U(1)$ charge of $\Psi$, must be related to the 'spin' J parametrizing the constraints via

$$
2 \mathrm{~J}-1=\frac{l-1}{n_{i}}-\frac{2 n_{i}}{l-1} .
$$

This illustrates the importance of introducing parameters into both the generators (1.1) and the ansatz (1.2) for the KP times. Moreover, we find that all the decoupling equations corresponding to each of the other primary fields in the correlator (1.4) are satisfied by virtue of the same set of Virasoro constraints, although the corresponding values of $l^{\prime}$ and $n_{i^{\prime}}$ would in general differ from those on the RHS of (1.5).

Reversing the argument, we can say that the correlators (1.4) solve the Virasoro constraints. This result can be considered as a variation of a general conclusion, drawn from Matrix Models, that Virasoro-constrained integrable hierarchies are related to minimal matter interacting with topological $2 d$ gravity?. An attempt towards understanding of the ansatz (1.4) in more general terms is given in the next subsection.

3 There is, in fact, a certain gap in the argument, as we do not actually prove the (Miwa-transformed) Hirota bilinear equations for the RHS of (1.4). What we can nevertheless show is that the decoupling equations satisfied by the correlator in (1.4) are consistent with the KP evolutions, which is a strong supporting argument in favour of the ansatz (1.4) for the tau function. 


\subsection{Solving Virasoro constraints by a 'target-space' theory}

The approach pursued in this paper is in a certain sense 'dual' to the usual way the time variables $t_{r}$ enter the theory. Recall [7, \&] that these are used as 'sources' or, 'external' parameters, to build up a generating function for the 'string' correlation functions in a topological gravity + matter theory:

$$
\log \tau(t) \sim \sum_{h} \sum_{N} \sum_{\left\{p_{a}\right\}}\left\langle\phi_{p_{1}} \ldots \phi_{p_{N}}\right\rangle_{h} t_{p_{1}} \ldots t_{p_{N}}
$$

(a formula of this type is actually valid for a KdV tau function, but we ignore such differences in this, very qualitative, discussion). On the other hand, the formulation which we develop below expresses the tau function as a single correlation function in what thus becomes an 'effective' theory in the sense that its genus-zero correlators provide an exact solution to the Virasoro constraints and thus account for the sum over all the genera of the 'string' correlation functions. The theory on the RHS of (1.4) might be thought of as living "in the $t$-space", or, in other words, the appropriate two-dimensional space is constructed out of the times/coupling constants/deformation parameter.

Although the worldsheet correlators involved in eq. (1.6) are those of a topological theory, we may try to understand the ansatz (1.4) qualitatively by considering for instance a more familiar object, (the generating function for) the tachyon scattering amplitudes summed over all genera [35]:

$$
\begin{aligned}
\tau[T] & =\sum_{h \geq 0} g^{2(h-1)}\left\langle e^{\int d^{2} \zeta T(X(\zeta))}\right\rangle_{h} \\
& =\sum_{h \geq 0} g^{2(h-1)} \sum_{N} \frac{1}{N !} \int d^{D} p_{1} \ldots \int d^{D} p_{N}\left\langle\prod_{a=1}^{N} \int d^{2} \zeta_{a} e^{i p_{a} X\left(\zeta_{a}\right)}\right\rangle_{h} \widetilde{T}\left(p_{1}\right) \ldots \widetilde{T}\left(p_{N}\right),
\end{aligned}
$$

where the brackets refer to a path integral in the theory with the free action

$$
S=\int d^{2} \zeta \partial X \bar{\partial} X
$$

\footnotetext{
${ }^{4}$ Note also the recent observations [33, 34] that the dependence of the structure functions on the deformation parameters in topological theories is governed by the same equations as those describing a space-time dependence of certain non-linear soluble systems.
} 
with the target-space indices suppressed (so, $p X=p_{\mu} X^{\mu}, \mu=1, \ldots, D$, etc.). The tachyon field $T$ thus plays the rôle of a set of 'external parameters'. Comparing with the ansatz (1.4), we see that for the (hypothetical) 'topological analogue' of (1.7) for $D \leq 2$ it would be possible to perform the sum over all the genera, which would suggest the 'effective' identifications

$$
t_{r} \sim \widetilde{T}(p)
$$

Moreover, the tree effective action corresponding to the theory (1.7), which is given as an integral over the $D$-dimensional target space, can be considered for $D=2$ as defining another conformal invariant theory. For $D=2$ (the $d=1$ string theory) there is just one propagating mode, the massless tachyon, and it is thus plausible that the quantum string theory loop expansion can be effectively represented by a quantum field theory, which (since $D=2$ and the metric is also quantized) can in fact be a conformal theory. The $\Psi_{j}$ from (1.4) are then to be thought of as operators of this theory, which must in principle be determined by the effective action'. Then, the ansatz (1.4) suggests that an appropriate 'topological version' of the above argument is apparently true for $d<1$ as well, with the effective theory being just the dressed minimal model (in fact, as we discuss later, essentially an $N=2$ minimal model!).

Also for $d=1$, the way the space-time dependence (i.e. that on the $z_{j}$ ) emerges can probably be considered along the lines of ref. [36], where two sets of times appeared, $t_{r}$ and $\bar{t}_{r}$, which leaves a possibility to express one of them via the $z_{j}$, as in (1.2), while viewing the other set as 'external' parameters. As a related problem, let us note that of the correspondence between the ansatz (1.4) and the standard 'fermionic' representation for the tau function: while the fermionic/Grassmannian construction of ref. [16] applies to a general tau function, eq. (1.4) necessarily gives a solution to the Virasoro constraints (and, at the same time, refers to a particular central charge- $d$ model). It may be expected that, for $d=1$ at least, bosonizing the matter (which would leave us with just two, the matter and the 'Liouville', scalars) would allow a 'decoupling' of one of the two scalars and refermionizing of the remaining one into the 'standard' fermions.

\subsection{Decoupling equations as BRST invariance}

Given a minimal model and wishing to relate it to the Virasoro constraints, we would have to dress it with an extra scalar in a particular way, called the 'Kontsevich-Miwa' dressing pre-

\footnotetext{
${ }^{5}$ We are grateful to R. Dijkgraaf and A. Tseytlin for important discussions of this point.
} 
scription, which would then allow us to interpret the $U(1)$ charges as the Miwa parameters and eventually recover the Virasoro constraints from the decoupling equations. An important point is that these latter must also be chosen in a special way from a family parametrized by the $U(1)$ charge of the primary field . These particular recipes, which make the decoupling equations amenable to the Kontsevich-Miwa transform, are in fact inherited from BRST invariance in a topological theory. Namely, the 'Kontsevich-Miwa' dressing prescription follows from the conditions defining chiral primary states [37] of the twisted $N=2$ ('topological') algebra [38, 39]. In addition, the particular decoupling equation to be chosen among a family of decoupling equations parametrized by the 'Liouville' charge, is determined just by the BRST-invariance condition in the topological algebra. Thus 'the' decoupling equations that are related to Virasoro constraints are essentially those of the $N=2$ model. We employ here the fact that any $d \leq 1 \cup d \geq 25$ matter can be embedded into a topological (twisted $N=2$ ) theory, according to a construction 40] of the topological algebra generators in terms of matter + 'Liouville' $+b c$ system fields. Unlike the case with the usual reparametrization ghosts, the $b$ field has conformal dimension 1 ๆ. We call this the 'mirror' BRST construction, in contrast with another one involving $c=-26$ reparametrization ghosts (see below). Then, the condition that the BRSTexact states be factored out from the representation of the topological algebra, boils down, in the matter + 'Liouville' sector, to the 'Kontsevich-Miwa'-dressed decoupling equations (i.e. precisely those decoupling equations that allow the Kontsevich-Miwa transform and thereby lead to the Virasoro constraints).

On the other hand, exploiting the other realization [40] of the topological algebra, which involves spin-2 $c=-26$ ghosts, allows us to recover the basic features of the DDK formalism. This can be summarized in a diagram,

\footnotetext{
${ }^{6}$ This choice does not mean any loss of generality; it specifies a particular 'lifting' of an arbitrary purelymatter null vector into a null vector in the tensor product theory.

7 So that, if one chooses to bosonize the matter, one would have two scalars with the opposite signatures and spin- $(1,0)$ ghosts, which constitute a topological sigma model in a flat $2 D$ target space.
} 


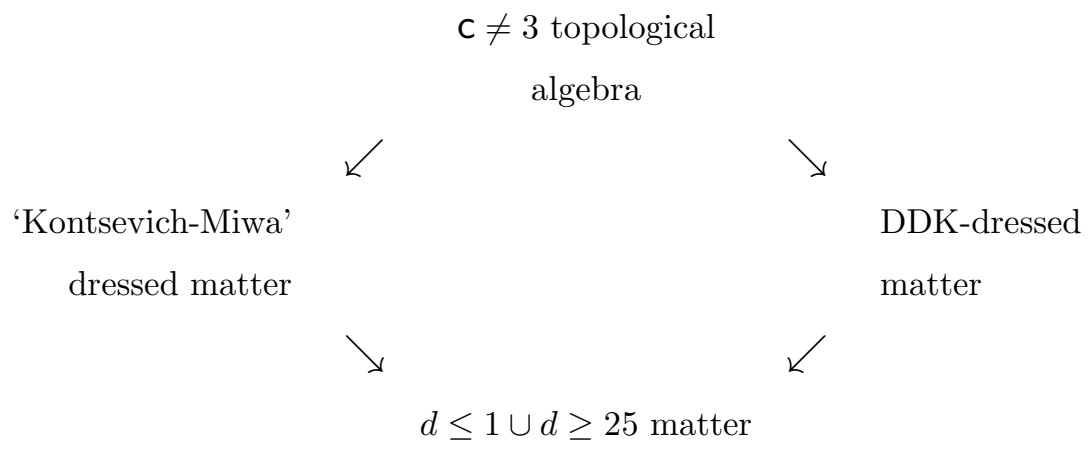

which applies to an arbitrary $d \leq 1 \cup d \geq 25$ matter theory and thus shows that any such theory can be embedded into a topological theory with topological central charge (or, the 'anomaly') $c \neq 3$. The lower arrows tell us that the matter part identified inside the matter + 'Liouville' theory is of course the same in both cases, as given by refs. [24, 43, 44] [. It appears that only the 'Kontsevich-Miwa' dressed version is related directly to the integrable formulation, while the two dressings should represent 'mirror' versions of the same theory; as we discuss in Sect. 3A, they both result from the two possible twistings of the proper $N=2$ algebra.

The matter central charge $d$ is given by

$$
d=\frac{(\mathrm{c}+1)(\mathrm{c}+6)}{\mathrm{c}-3}
$$

in terms of the topological central charge c, and therefore the restrictions $d \leq 1$ or $d \geq 25$ might be viewed as a result of the 'breakdown' of the twisted $N=2$ symmetry. The standard formula for the dimension of the $(l, 1)$ highest-weight states then follows from the $N=2$ machinery as

$$
\delta^{(l)}=-\frac{\left(l^{2}-1\right)}{4}\left(\frac{\mathrm{c}-3}{6}\right)^{ \pm 1}+\frac{1-l}{2}=\frac{13-d \pm \sqrt{(1-d)(25-d)}}{48}\left(l^{2}-1\right)+\frac{1-l}{2} .
$$

Note also that as c grows from $-\infty$ to 3 and from 3 to $+\infty$, each of the allowed values of $d$ is taken twice, except for the extrema of the function (1.9), $(\mathrm{c}=-3, d=1)$ and $(\mathrm{c}=9, d=25)$.

8 The issue of interpreting matter theories together with the corresponding ghosts as topological theories was addressed probably for the first time in [42]. The relation [40 between the twisted $N=2$ theories and the ordinary matter, as given by the two right arrows in (1.8), has recently received in 53 a powerful generalization to the case of W matter. It is a particular case of the equivalence of categories investigated in 54 .

9 The null vectors in the matter sector (without dressing) are of course the standard ones [23, 24]. Thus the $U(1)$ current might be considered superficial, as soon as it cannot affect the 'dynamical' content of the story, which can only be based on the Virasoro null vectors. This is, however, precisely what we mean by dressing: the rôle of the $U(1)$ current is to rearrange the standard decoupling equations so as to make them amenable to the Kontsevich-Miwa transform; the current does this job 'uniformly' for different-level decoupling equations. 
Thus the topological central charge provides a 'two-sheeted covering' of the allowed region of the matter central charge.

By topological central charge we mean here the parameter (the true central charge of the untwisted $N=2$ algebra 38, 39]) appearing in the topological algebra

$$
\begin{array}{llrl}
{\left[\mathcal{L}_{m}, \mathcal{L}_{n}\right]} & =(m-n) \mathcal{L}_{m+n}, & {\left[\mathcal{H}_{m}, \mathcal{H}_{n}\right]=\frac{\mathrm{c}}{3} m \delta_{m+n, 0},} & \\
{\left[\mathcal{L}_{m}, \mathcal{G}_{n}\right]} & =(m-n) \mathcal{G}_{m+n}, & {\left[\mathcal{H}_{m}, \mathcal{G}_{n}\right]=\mathcal{G}_{m+n},} & \\
{\left[\mathcal{L}_{m}, \mathcal{Q}_{n}\right]} & =-n \mathcal{Q}_{m+n}, & {\left[\mathcal{H}_{m}, \mathcal{Q}_{n}\right]=-\mathcal{Q}_{m+n},} & \\
{\left[\mathcal{L}_{m}, \mathcal{H}_{n}\right]} & =-n \mathcal{H}_{m+n}+\frac{\mathrm{c}}{6}\left(m^{2}+m\right) \delta_{m+n, 0}, & & \\
\left\{\mathcal{G}_{m}, \mathcal{Q}_{n}\right\} & =2 \mathcal{L}_{m+n}-2 n \mathcal{H}_{m+n}+\frac{\mathrm{c}}{3}\left(m^{2}+m\right) \delta_{m+n, 0},
\end{array}
$$

We thus establish a path leading from (1.11) to the Virasoro constraints (1.1). The relation between, say, $\mathcal{L}_{n}$ and $\mathrm{L}_{p}$ appears not quite obvious. In particular, we will see that (for c $<3$, for instance) the respective parameters are related via $\mathrm{J}=\frac{1}{2}+\frac{1}{2} \sqrt{\frac{3-\mathrm{c}}{3}}-\sqrt{\frac{3}{3-\mathrm{c}}}$. We will proceed in two steps, by first reducing from the BRST-exact topological states to the 'Kontsevich-Miwa' dressed decoupling equations, and then extracting from the latter the Virasoro constraints.

The fact that it is essentially the $N=2$ symmetry that leads to both the DDK formalism and the Virasoro constraints, suggests regarding it as a certain unifying notion. In a worldsheet phase the $N=2$ generators split into reparametrization ghosts plus matter (including the Liouville), while a different splitting involving $c=-2$ ghosts (cf. [45]) is related to the 'integrable' formulation. The two pictures differ by choosing one out of the two possible twistings of the proper $N=2$ algebra 52 .

It was first observed in 21] that the level-2 decoupling equations essentially coincide with the Virasoro constraints (1.3) in which the background charge $2 \mathrm{~J}-1$ is given by eq. (1.5) for $l=2$. The level- $l$ generalization, as given by (1.5), was further suggested in [46]. However, the interpretation given there of the Kontsevich-Miwa transformed level-3 decoupling equation requires being corrected. The actual mechanism underlying the correspondence between Virasoro constraints and the decoupling equations has proved to consist in the factorization of the decoupling equations through the Virasoro generators (1.1) with the appropriate background charge (1.5). 
This paper is organized as follows. In Sect. 2, we review the issue of the Virasoro-constrained KP hierarchy and give a preliminary analysis, to be considered as a motivation (or, as an 'elementary explanation') for the subsequent use of the Kontsevich-Miwa transform, which relates the 'integrable' formalism to the conformal field-theoretic data. As a 'toy' case, we subject the tau function to only the $L_{ \pm 1}$ and $L_{0}$ constraints, and show that these are precisely the projective Ward identities of the corresponding conformal theory, provided the parameters involved satisfy certain relations. Starting with Sect. 3, we adopt as a 'first principle' the notion of twisted $N=2$ symmetry and show how the associated BRST-invariance condition gives rise to exactly the decoupling equations that turn into the Virasoro constraints via the KontsevichMiwa transform. To 'legitimate' taking the topological algebra as a starting point, we show in the Appendix to Sect. 3 that the other reduction of the topological algebra gives the standard DDK formalism. The dressed decoupling equations that follow from the BRST-invariance condition for ghost-independent correlators can also be constructed directly, by imposing certain restrictions on the general tensor product null vectors. This is considered in Sect. 4. Further, in Sect. 5 we show that the Kontsevich-Miwa transform leads to a reformulation of the decoupling equations in terms of Virasoro constraints. In Sect. 6, we suggest a generalization of the lowest-l cases to arbitrary $l$. Sect. 7 contains several concluding remarks and an outlook.

\section{Constrained KP hierarchy and the Kontsevich-Miwa transform}

\section{$2.1 \quad$ KP hierarchy}

Let us start with the KP hierarchy [16]. It can be described either in the evolutionary form, as an infinite set of equations on (coefficients of) a pseudodifferential operator, or as (Hirota) bilinear relations on the tau function. The tau-functional description may be considered as having the more direct relevance to 'physics', being related to the partition function, while the

evolutionary form has all the usual advantages due to the introduction of a spectral parameter and the associated wave function. The wave function depends on the spectral parameter $z$ via

$$
\begin{gathered}
\psi(t, z) \equiv e^{\xi(t, z)} w(t, z)=e^{\xi(t, z)} \frac{\tau\left(t-\left[z^{-1}\right]\right)}{\tau(t)} \\
\xi(t, z)=x z+\sum_{r \geq 1} t_{r} z^{r}
\end{gathered}
$$


where

$$
t \pm\left[z^{-1}\right]=\left(t_{1} \pm z^{-1}, t_{2} \pm \frac{1}{2} z^{-2}, t_{3} \pm \frac{1}{3} z^{-3}, \ldots\right)
$$

(the plus signs are encountered in the adjoint wave function). Here $t=\left(t_{1}, t_{2}, t_{3}, \ldots\right)$ are the time parameters of the hierarchy. Knowing the wave function $w(t, z)$ allows us to construct the dressing operator $K$ as

$$
w(t, z)=1+\sum_{n \geq 1} w_{n}(t) z^{-n} \quad \Longrightarrow \quad K=1+\sum_{n \geq 1} w_{n}(t) D^{-n}, \quad D \equiv \frac{\partial}{\partial x}
$$

Now the evolution equations on $K$ read

$$
\frac{\partial}{\partial t_{r}} K=-\left(K D^{r} K^{-1}\right)_{-} K, \quad r \geq 1
$$

The above form of $z$ dependence, eq. (2.2), is 'simulated' by the Kontsevich-Miwa parametrization (1.2) for the times $t_{r}$; its heuristic similarity to (2.2) may be considered as an 'explanation' of its viability. Below, the Miwa parameters $n_{j}$ will acquire the rôle of 'Liouville' $U(1)$ charges of field operator insertions sitting at the points $z_{j}$.

\section{$2.2 \quad$ Virasoro constraints}

As was noted in the Introduction, we will consider, on the KP hierarchy, more general constraints than those that have actually been derived from specific matrix models, by allowing the constraints to depend on a parameter J. That is, we introduce the constraints

$$
\mathrm{L}_{p} \tau=0, \quad p \geq-1
$$

with the Virasoro generators $\mathrm{L}_{p}$ as given in eq. (1.1). The parameter $\mathrm{J}$, which would have parametrized the central charge as $-2\left(6 \mathrm{~J}^{2}-6 \mathrm{~J}+1\right)$, had the $\mathrm{L}_{\leq-2}$ generators been involved as well, can be thought of as the 'spin' (dimension) of an abstract bc system underlying the Virasoro generators, $\sum_{n \in \mathbf{Z}} \mathrm{L}_{n} z^{-n-2} \sim(1-\mathrm{J}) \partial b c-\mathrm{J} b \partial c$.

That the action on the KP hierarchy via the generators (1.1) is compatible with the KP flows 
has been proved in [47] (see also [48] and references therein). Indeed, when the infinitesimal action $\tau \mapsto \mathrm{L}_{n} \tau$ is translated into an action on dressing operators, it becomes

$$
\begin{gathered}
K \mapsto \mathbf{L}_{p} K \equiv\left(K\left(\mathrm{~J}(p+1) D^{p}+P D^{p+1}\right) K^{-1}\right)_{-} K \\
P \equiv x+\sum_{r \geq 1} r t_{r} D^{r-1}=x+t_{1}+2 t_{2} D+\ldots
\end{gathered}
$$

It is now completely straightforward to check [47] the compatibility of this action with the flows (2.4), which holds irrespectively of the value of J. Hence follows, in particular, the consistency of the hierarchy flows with the Virasoro constraints.

Note that eq. (1.2) allows us to rewrite the Virasoro generators $\mathbf{L}_{p}$ as

$$
\mathbf{L}_{p}=\left(K\left(\mathrm{~J}(p+1) D^{p}+x D^{p+1}+\sum_{j} \frac{n_{j}}{z_{j}-D} D^{p+1}\right) K^{-1}\right)_{-}
$$

and therefore the constraint

$$
\sum_{p \geq-1} \mathbf{L}_{p} z^{-p-2}=0
$$

takes a rather suggestive formएण

$$
\left(K\left(-\mathrm{J} \frac{\partial}{\partial z}+x+\sum_{j} \frac{n_{j}}{z_{j}-D}\right) \frac{1}{z-D} K^{-1}\right)_{-}=0 .
$$

\subsection{Why the Kontsevich-Miwa transform?}

It will be shown in the subsequent sections that the Virasoro constraints (2.5) can be solved by substituting for the tau function, considered as a function of the $z_{j}$, the ansatz

$$
\tau\left\{z_{j}\right\}=\lim _{N \rightarrow \infty}\left\langle\Psi_{1}\left(z_{1}\right) \ldots \Psi_{N}\left(z_{N}\right)\right\rangle
$$

\footnotetext{
${ }^{10}$ From the results of [6] we know that the quasiclassical ('dispersionless' [49, 50]) limit of (2.7) or (2.9) would give a reformulation of the Virasoro constraints in the Landau-Ginzburg theory. Then, by the construction of the present paper, which relates the Virasoro constraints to the (essentially $N=2$ ) decoupling equations, the resulting 'quasiclassical' equation should be a Landau-Ginzburg reformulation of the decoupling equations of a (twisted) $N=2$ model.
} 
with \langle\rangle and the $\Psi_{j}$ being, respectively, the chiral correlation function and primary field operators in a conformal field theory on the $z$ plane. These CFT ingredients will be constructed systematically in the subsequent sections, while now we would like to consider, as a motivation, a 'toy' case involving only three constraints, $L_{-1}, L_{0}$ and $L_{1}$. This would already allow us to see how the standard CFT notions can enter the game.

The $\Psi_{j}$ operators will be identified as those of a minimal conformal model tensored with an extra $U(1)$ current $I$. The rôle and the origin of the current will be discussed below in some detail, but all we need to know at the moment is that the Virasoro generators can be chosen in such a way that there is a zero background charge in the $U(1)$ sector, and therefore the correlators of $\exp \beta_{j} \phi\left(z_{j}\right), \phi(z) \sim \int^{z} I$, can be evaluated easily. Moreover, it turns out that the exponents $\beta_{j}$ required by the dressing prescription must be equal to the $n_{j}$. Thus,

$$
\left\langle\prod_{j} \Psi_{j}\left(z_{j}\right)\right\rangle=\prod_{k<l}\left(z_{k}-z_{l}\right)^{-n_{k} n_{l}}\left\langle\prod_{j} \psi_{j}\left(z_{j}\right)\right\rangle,
$$

where the $\psi_{j}$ should pertain to a minimal conformal model. Recall further the 'projective Ward identities' 24]

$$
\begin{array}{r}
\sum_{j}\left(z_{j}^{p+1} \frac{\partial}{\partial z_{j}}+(p+1) z_{j}^{p} \delta_{j}\right)\left\langle\prod_{j} \psi_{j}\left(z_{j}\right)\right\rangle=0 \\
p=-1,0,1
\end{array}
$$

where $\delta_{j}$ is the conformal dimension of $\psi_{j}$. This gives

$$
\begin{array}{r}
\sum_{j}\left(z_{j}^{p+1} \frac{\partial}{\partial z_{j}}+\frac{1}{2} \sum_{k \neq j} n_{j} n_{k} \frac{z_{j}^{p+1}-z_{k}^{p+1}}{z_{j}-z_{k}}+(p+1) z_{j}^{p} \delta_{j}\right)\left\langle\prod_{j} \Psi_{j}\left(z_{j}\right)\right\rangle=0 \\
p=-1,0,1 .
\end{array}
$$

It turns out that these equations are nothing but the three Virasoro constraints if we use the Kontsevich-Miwa parametrization (1.2) 凹: For $p=-1$, quite simply, the operator in (2.13) is just

\footnotetext{
${ }^{11}$ The fact that the operators on the LHS of eqs. (2.13) form an $s l_{2}$ algebra is of course quite trivial; less obvious, however, is that transforming the 'projective' $\mathrm{sl}(2)$ generators into the time variables allows us to anticipate the relations, such as eq. (2.16), between the different parameters, to be derived systematically below from the analysis of the decoupling equations.
} 


$$
\sum_{j} \frac{\partial}{\partial z_{j}}=-\sum_{j} n_{j} \sum_{r \geq 1} z_{j}^{-r-1} \frac{\partial}{\partial t_{r}}=-\sum_{r \geq 1}(r+1) t_{r+1} \frac{\partial}{\partial t_{r}} \equiv-\mathrm{L}_{-1}
$$

Similarly, for $p=0$ we get

$$
\begin{aligned}
& -\sum_{j} z_{j} n_{j} \sum_{r \geq 1} z_{j}^{-r-1} \frac{\partial}{\partial t_{r}}+\frac{1}{2} \sum_{j} \sum_{k \neq j} n_{j} n_{k}+\sum_{j} \delta_{j} \\
& =-\sum_{r \geq 1} r t_{r} \frac{\partial}{\partial t_{r}}+\frac{1}{2}\left(\sum_{j} n_{j}\right)^{2}+\sum_{j}\left(\delta_{j}-\frac{n_{j}^{2}}{2}\right) \\
& =-\sum_{r \geq 1} r t_{r} \frac{\partial}{\partial t_{r}} \equiv-\mathrm{L}_{0} .
\end{aligned}
$$

The 'unwanted' terms here have been cancelled by imposing the relation

$$
\delta_{j}=\frac{1}{2} n_{j}^{2}-\frac{1}{2} Q n_{j}
$$

with $Q=\sum_{j} n_{j}$ (we will derive these relations in a more systematic way in (6.4); for a given $\delta_{j}$, eq. (2.16) allows us to determine the corresponding $n_{j}$ ). And finally, for $p=1$ we obtain the operator

$$
\begin{aligned}
& -\sum_{j} n_{j} \sum_{r \geq 0} z_{j}^{-r} \frac{\partial}{\partial t_{r+1}}+\frac{1}{2} \sum_{j} \sum_{k \neq j} n_{j} n_{k}\left(z_{j}+z_{k}\right)+2 \sum_{j} z_{j} \delta_{j} \\
& =-\sum_{j} n_{j} \frac{\partial}{\partial t_{1}}-\sum_{r \geq 1} r t_{r} \frac{\partial}{\partial t_{r+1}}+\sum_{j} n_{j} z_{j}\left(Q-n_{j}\right)+2 \sum_{j} z_{j} \delta_{j} \\
& =-\sum_{r \geq 1} r t_{r} \frac{\partial}{\partial t_{r+1}}-Q \frac{\partial}{\partial t_{1}} \equiv-\mathrm{L}_{1},
\end{aligned}
$$

from which we see that $Q$ takes the rôle of the background charge underlying the Virasoro generators (1.1),

$$
Q=\mathrm{Q} \equiv 2 \mathrm{~J}-1
$$

The above should be viewed merely as a motivation for using the Kontsevich-Miwa transform and the ansatz (2.10) for the tau function. It is significant, however, that already such a generic property of conformal models as the projective invariance, is captured by the standard $\mathrm{L}_{ \pm 1}$, 
$\mathrm{L}_{0}$ constraints in terms of the times $t_{r}$, with the parameters involved being related as in eqs. (2.10), 2.18).

The rest of the Virasoro constraints $L_{\geq 2}$ no longer follow from the projective invariance of CFT, but rather from the decoupling equations, which contain the dynamical information about a particular minimal model. The actual derivation of the Virasoro constraints from the decoupling equations will be considered in Sect. 5 .

Searching for a most systematic approach, we will switch in the next section to the topological algebra. It is from the BRST invariance in its highest-weight representations that we will be able to recover at the end the (specially dressed) decoupling equations, and then the Virasoro constraints (2.5). The above relations (2.16) and (2.18) will reappear in the course of the derivation.

\section{From the twisted $N=2$ and BRST invariance to dressed null states}

We start in this section with a construction of the topological algebra (1.11), and then use this construction to 'reduce' the requirement of BRST invariance, taken in the form of factoring out BRST-trivial states, to the dressed decoupling equations. The construction of the algebra itself is level-independent, while the reduction has to be considered level by level. To legitimate taking the topological algebra as our starting point, we show in the Appendix to this section that the $N=2$ symmetry may claim the rights of the DDK formulation: in fact, the topological algebra admits a reduction to the DDK formalism and thus can be viewed as a generalization

of it. We also comment in the Appendix on the relation between the two constructions of the topological algebra. Only one of these will be used in the rest of the paper, and it will eventually lead us, via the Kontsevich-Miwa transform, to the Virasoro constraints.

\subsection{A 'mirror' BRST construction for the topological algebra}

The fields used to construct our specific realization of the topological algebra include matter, an additional $U(1)$ current $I$, which will be referred to as the 'Liouville' current, and a $c=-2$ $b c$ system. This spin-1 (anticommuting) bc system (the $\eta \xi$ system, 41]) is defined by 


$$
\begin{aligned}
& b(z)=\sum_{n \in \mathbf{Z}} b_{n} z^{-n-1}, \quad c(z)=\sum_{n \in \mathbf{Z}} c_{n} z^{-n}, \\
& \left\{b_{n}, c_{m}\right\}=\delta_{m+n, 0}, \quad b_{\geq 0}|0\rangle_{\mathrm{gh}}=c_{>0}|0\rangle_{\mathrm{gh}}=0 .
\end{aligned}
$$

The matter Virasoro generators combine with the Sugawara 'Liouville' contribution $-\frac{1}{2} \sum_{n \in \mathbf{Z}}: I_{m-n} I_{n}:$ into the generators $L_{m}$ which satisfy

$$
\begin{aligned}
{\left[L_{m}, L_{n}\right] } & =(m-n) L_{m+n}+\frac{d+1}{12}\left(m^{3}-m\right) \delta_{m+n, 0} \\
{\left[L_{m}, I_{n}\right] } & =-n I_{m+n} \\
{\left[I_{m}, I_{n}\right] } & =-m \delta_{m+n, 0}
\end{aligned}
$$

where the 1 in the central charge $d+1$ is the $U(1)$ contribution. We find it convenient to 'twist' the Virasoro generators as

$$
\widehat{L}_{m}=L_{m}+\frac{1}{2} Q(m+1) I_{m}
$$

where $Q$ is the matter background charge

$$
Q=\sqrt{\frac{1-d}{3}}
$$

which therefore becomes also that of the 'Liouville' scalar. Then, the central charge of the new Virasoro generators is just $1-3 Q_{\mathrm{m}}^{2}+1+3 Q_{\mathrm{L}}^{2}=2$, so that the corresponding formulae in (3.2) get replaced by

$$
\begin{aligned}
{\left[\widehat{L}_{m}, \widehat{L}_{n}\right] } & =(m-n) \widehat{L}_{m+n}+\frac{2}{12}\left(m^{3}-m\right) \delta_{m+n, 0} \\
{\left[\widehat{L}_{m}, I_{n}\right] } & =-n I_{m+n}-\frac{1}{2} Q\left(m^{2}+m\right) \delta_{m+n, 0}
\end{aligned}
$$

Now, centreless Virasoro generators as those in (1.11) can be constructed by adding the $c=-2$ ghost contribution:

$$
\mathcal{L}_{m}=\widehat{L}_{m}+l_{m}, \quad l_{m}=\sum_{n \in \mathbf{Z}} n: b_{m-n} c_{n}:
$$

[the ghost energy-momentum tensor being $\left.T_{\mathrm{gh}}(z)=-: b \partial c:(z)\right]$. Further, introducing the ghost current $i=-: b c$ : we define the topological $U(1)$ current as 


$$
\mathcal{H}_{m}=i_{m}+\sqrt{\frac{3-\mathrm{c}}{3}} I_{m}
$$

so that

$$
\left[\mathcal{H}_{m}, \mathcal{H}_{n}\right]=\frac{\mathrm{c}}{3} m \delta_{m+n, 0}
$$

It follows that the topological algebra commutator

$$
\left[\mathcal{L}_{m}, \mathcal{H}_{n}\right]=-n \mathcal{H}_{m+n}+\frac{\mathrm{c}}{6}\left(m^{2}+m\right) \delta_{m+n, 0}
$$

can now be established provided the matter central charge $d$ is related to the topological central charge c by eq. (1.9).

Next, we need to construct the remaining generators of the topological algebra (1.11). As the ghost field $b$ is of dimension 1 , the ansatz for the BRST current $\mathcal{Q} \sim c T$, which works for $c$ being of dimension -1 (see the Appendix to this section) does not apply here. Instead, we can identify the modes of a spin-1 odd current $\mathcal{Q}(z)$ simply as

$$
\mathcal{Q}_{m}=b_{m}
$$

On the other hand, it is the spin-2 fermionic field $\mathcal{G}(z)$ that now comprises the 'non-trivial' terms, usually characteristic to the BRST generators when these are built 44] using a spin-2 $b$ field:

$\mathcal{G}_{m}=2 \sum_{p \in \mathbf{Z}} c_{m-p} \widehat{L}_{p}+2 \sqrt{\frac{3-\mathrm{c}}{3}} \sum_{p \in \mathbf{Z}}(m-p) c_{m-p} I_{p}+\sum_{p, r \in \mathbf{Z}}(r-p): b_{m-p-r} c_{r} c_{p}:+\frac{\mathrm{c}}{3}\left(m^{2}+m\right) c_{m}$

To avoid misunderstanding, let us stress that the $\widehat{L}_{p}$ generators here include, besides the 'improvement' term written out explicitly in (3.3), also the Sugawara 'Liouville' contribution $-\frac{1}{2} I^{2}$. Thus,

$$
\widehat{L}_{p}=\bar{L}_{p}-\frac{1}{2} \sum_{n}: I_{p-n} I_{n}:+\frac{1}{2} Q(p+1) I_{p}
$$


where the $\bar{L}_{p}$ are purely-matter Virasoro generators, with the central charge given by (1.9).

The coefficient in front of the second term in (3.11) (and the same coefficient in (3.7)) is real for $\mathrm{c}<3$; the regions $\mathrm{c}<3$ and $\mathrm{c}>3$ are 'mirrored' in that the matter and the 'Liouville' take the place of each other: all the coefficients in the above ansatze for the topological algebra generators can be kept real when going over from $c<3$ to $c>3$, by reversing the signature of the fields.

All the commutation relations (1.11) can now be verified straightforwardly. We have thus arrived at a realization of the topological algebra (1.11) and the relation (1.9) between the central charges. It is valid, irrespective of whatever $d \leq 1$ or $d \geq 25$ matter theory is taken at the start [2]. Below, we will use this realization in order to translate the BRST-invariance condition into the matter + 'Liouville' theory.

\subsection{Decoupling equations from BRST invariance}

Let us consider a representation of the topological algebra built on a BRST-invariant chiral primary state $|\Phi\rangle$ 37]

$$
\begin{aligned}
\mathcal{Q}_{0}|\Phi\rangle & =0, \\
\mathcal{L}_{\geq 0}|\Phi\rangle & =\mathcal{H}_{\geq 1}|\Phi\rangle=\mathcal{G}_{01}|\Phi\rangle=0 \\
\mathcal{H}_{0}|\Phi\rangle & =\mathrm{h}|\Phi\rangle .
\end{aligned}
$$

The topological $U(1)$ charge $\mathrm{h}$ is thus the only non-zero parameter that distinguishes between such states. The dimension zero condition will translate below into the 'Kontsevich-Miwa' dressing condition, while a 'level-by-level' imposition of the BRST invariance in the above representations will give rise to the particular decoupling equations that are amenable to the Kontsevich-Miwa transform. Namely, provided $\mathrm{h}$ is related to $\mathrm{c}$ by a certain quadratic equation [see (6.11) for the $(l, 1)$ case], there will be primary states which are BRST-exact and should therefore be factored out. This is achieved by imposing equations, analogous to the decoupling ones, which for the ghost-independent [and hence, in view of (3.10), BRST-invariant] insertions boil down to the 'Kontsevich-Miwa' dressed decoupling equations. These latter will thus be derived in the remaining part of this section, while in Sect. 5 their relation to the Virasoro

\footnotetext{
${ }^{12}$ Note in particular that there is no need to bosonize the matter; however, when explicitly bosonizing it, a similar construction (actually, the one discussed in the Appendix to this section) can also be arrived at via the hamiltonian reduction $[53]$.
} 
constraints will be demonstrated.

\subsubsection{Level-2 reduction and the decoupling equation}

We start with the simplest, level-2, case of imposing the BRST-invariance condition in a representation of the topological algebra (1.11), to show that under the splitting, as in Sect. 3.1, of a BRST-invariant highest-weight state into matter $\otimes$ 'Liouville' $\otimes$ ghosts, the matter $\otimes$ 'Liouville' part becomes a null vector dressed in such a way that it allows a subsequent application of the Kontsevich-Miwa transform.

The BRST-invariant states, $\mathcal{Q}_{0}|\Xi\rangle=0$, at level 2 are of the form

$$
|\Xi\rangle=\left(\alpha \mathcal{L}_{-1}^{2}+\mathcal{L}_{-2}+\Gamma \mathcal{H}_{-1} \mathcal{L}_{-1}+\frac{1}{2} \Gamma \mathcal{Q}_{-1} \mathcal{G}_{-1}\right)|\Phi\rangle,
$$

with $\alpha$ and $\Gamma$ arbitrary so far. A crucial point is that the BRST invariance rules out the $\mathcal{H}_{-2}$ and $\mathcal{H}_{-1}^{2}$ terms. Later, we will see that this property, which persists to higher levels, can be put into the basis of an independent 'direct' derivation of the desired dressed null vectors.

Impose further on $|\Xi\rangle$ the highest-weight conditions w.r.t. the topological algebra. From $\mathcal{G}_{1}|\Xi\rangle=0$ we find

$$
2 \alpha+3+\Gamma\left(\mathrm{h}+1+\frac{\mathrm{c}}{3}\right)=0 .
$$

Further, the constraint $\mathcal{H}_{1}|\Xi\rangle=0$ gives two equations

$$
\left\{\begin{array}{l}
2 \alpha \mathrm{h}+\Gamma\left(\frac{\mathrm{c}}{3}-1\right)=0 \\
1+\Gamma \mathrm{h}=0
\end{array}\right.
$$

All the highest-weight conditions

$$
\mathcal{Q}_{0}|\Xi\rangle=\mathcal{Q}_{\geq 1}|\Xi\rangle=\mathcal{G}_{\geq 1}|\Xi\rangle=\mathcal{L}_{\geq 1}|\Xi\rangle=\mathcal{H}_{\geq 1}|\Xi\rangle=0
$$

are now satisfied. Indeed, the $\mathcal{Q}_{1^{-}}$and $\mathcal{G}_{2}$-conditions follow from the established ones via $\mathcal{Q}_{1}=\left[\mathcal{Q}_{0}, \mathcal{H}_{1}\right], \mathcal{G}_{2}=\left[\mathcal{H}_{1}, \mathcal{G}_{1}\right]$. Further, $\mathcal{L}_{1}=\frac{1}{2}\left\{\mathcal{G}_{1}, \mathcal{Q}_{0}\right\}$ follows as well, and therefore, via $\left[\mathcal{L}_{1}, \mathcal{G}_{n}\right]=(1-n) \mathcal{G}_{n+1}$ and $\left[\mathcal{L}_{1}, \mathcal{Q}_{n}\right]=-n \mathcal{Q}_{n+1}$, so do $\mathcal{G}_{\geq 3}$ and $\mathcal{Q}_{\geq 2}$. Generated similarly are 
the $\mathcal{H}_{\geq 2}$ conditions. Finally, $\left\{\mathcal{G}_{1}, \mathcal{Q}_{1}\right\}=2 \mathcal{L}_{2}-2 \mathcal{H}_{2}$, which implies the $\mathcal{L}_{2}$ and hence all the $\mathcal{L}_{\geq 2}$ conditions.

It follows that

$$
2 \mathrm{~h}^{2}-\mathrm{h}\left(\frac{\mathrm{c}}{3}+1\right)+\frac{\mathrm{c}}{3}-1=0
$$

from which we find two solutions

$$
\mathrm{h}=\left\{\begin{array}{l}
\frac{\mathrm{c}-3}{6} \\
1
\end{array}, \quad \alpha=\left\{\begin{array}{l}
\frac{6}{\mathrm{c}-3} \\
\frac{\mathrm{c}-3}{6}
\end{array}, \quad \Gamma=\left\{\begin{array}{l}
\frac{6}{3-\mathrm{c}} \\
-1
\end{array}\right.\right.\right.
$$

Now, the state $|\Xi\rangle$ thus constructed proves to be not only BRST-invariant, but also BRSTexact:

$$
|\Xi\rangle=\mathcal{Q}_{0}\left(\frac{\alpha}{2} \mathcal{L}_{-1} \mathcal{G}_{-1}+\frac{1}{2} \mathcal{G}_{-2}+\frac{1}{2} \Gamma \mathcal{H}_{-1} \mathcal{G}_{-1}\right)|\Phi\rangle .
$$

Such states should be factored out, which can be accomplished by imposing certain equations on the correlation functions involving $\Phi$ (in obvious analogy with the use of decoupling equations to factor away null vectors from Verma modules [24, 44]).

At this point we introduce into our scheme the Miwa parameter $\hat{n} \equiv n_{i}$ as the 'Liouville' charge of $\Phi\left(z_{i}\right)$, according to (3.7): we set

$$
\mathrm{h}=\sqrt{\frac{3-\mathrm{c}}{3}} \hat{n} .
$$

Then from eq. (3.18) $\hat{n}$ is given in terms of the topological central charge, and hence in terms of the matter central charge, by

$$
\hat{n}=\left\{\begin{array}{l}
-\frac{1}{2} \sqrt{\frac{3-c}{3}} \\
\sqrt{\frac{3}{3-c}}
\end{array} \quad \Longrightarrow \quad \hat{n}^{2}=\frac{13-d \pm \sqrt{(1-d)(25-d)}}{24} .\right.
$$


Now, to perform the reduction to the matter $\otimes$ 'Liouville' theory, we substitute for the topological generators the expressions (3.6), (3.7), (3.10), and (3.11), and also take

$$
|\Phi\rangle=|\Psi\rangle \otimes|0\rangle_{\mathrm{gh}}
$$

This form of $|\Phi\rangle$, with no ghost oscillators involved, means in particular that the state is BRST invariant, $b_{0}|\Phi\rangle=0$. Then, using (3.1) and (3.19), we find that [for both the upper and the lower cases in (3.19), (3.22)]:

$$
|\Xi\rangle=|\Upsilon\rangle \otimes|0\rangle_{\mathrm{gh}}
$$

where

$$
\begin{aligned}
|\Upsilon\rangle & =\left(\alpha \widehat{L}_{-1}^{2}+\widehat{L}_{-2}+\gamma I_{-1} \widehat{L}_{-1}\right)|\Psi\rangle \\
& =\left(\alpha L_{-1}^{2}+L_{-2}+\beta I_{-2}+\gamma I_{-1} L_{-1}\right)|\Psi\rangle \\
\alpha & =-\frac{1}{2 \hat{n}^{2}}, \quad \beta=\hat{n}-\frac{1}{2 \hat{n}}, \quad \gamma=-\frac{1}{\hat{n}} .
\end{aligned}
$$

The two expressions for $|\Upsilon\rangle$ are related by eq. (3.3), in which $Q=\frac{1}{\hat{n}}-2 \hat{n}$, as can be found by comparing eqs. (3.22) and (3.4).

We have thus obtained a null vector in the matter theory tensored with the 'Liouville': $L_{\geq 1}|\Upsilon\rangle=I_{\geq 1}|\Upsilon\rangle=0$. To get down to the bottom of the diagram (1.8), we subtract away from the Virasoro generators $L_{n}$ the Sugawara 'Liouville' contribution, and in this way recover the usual 'minimal' null vector in the $(d, \delta)$ Verma module, with the central charge $d$ given by (1.9). As for the highest weight $\delta$, it is found to be

$$
\delta=\left\{\begin{array}{l}
-\frac{c}{8}-\frac{1}{8} \\
-\frac{c+6}{2(c-3)}
\end{array}=\frac{5-d \pm \sqrt{(1-d)(25-d)}}{16}\right.
$$

which is a standard expression.

At the level of correlation functions, the condition for $|\Upsilon\rangle$ to factor out takes the form of a decoupling equation for correlators of the form 


$$
\left\langle\Psi\left(z_{i}\right) \prod_{j \neq i} \Psi_{j}\left(z_{j}\right)\right\rangle
$$

Obviously, at least one operator insertion here must be that of $\Psi$, and thus the corresponding insertion point $z_{i}$ will from now on be singled out from the rest of the $z_{j}$. The other insertions, of dimensions ${ }^{\text {t3 }} \Delta_{j}$ and $U(1)$ charges $n_{j}$, may or may not coincide with $\Psi$.

In order to derive the decoupling equations from the conditions $\left\langle\Upsilon\left(z_{i}\right) \prod_{j \neq i} \Psi_{j}\left(z_{j}\right)\right\rangle=0$, we proceed in the standard way [24], but in addition restrict ourselves to a subspace of those operators $\Psi_{j}$ whose dimensions and $U(1)$ charges are related by the 'Kontsevich-Miwa' dressing condition:

$$
\Delta_{j}=-\frac{1}{2} Q n_{j} \stackrel{(l=2)}{=}\left(\hat{n}^{2}-\frac{1}{2}\right) \frac{n_{j}}{\hat{n}}
$$

From the point of view of the matter + 'Liouville' theory, this dressing condition just says that the scalar $\phi(z) \sim \int^{z} I$ enters vertex operators with an exponent determined by the matter part. However, we also know the 'invariant' meaning of (3.28), which is tantamount to saying that the -dimension of $\Psi_{j}$ is zero, and therefore, for ghost-independent insertions, so is the 'topological' $\mathcal{L}$-dimension; thus the $\Psi_{j}$ are ghost-independent representatives of chiral primary fields. In fact, the dressing condition (3.28) is the same as (2.16), with $\Delta_{j}$ the sum of the matter dimension $\delta_{j}$ and the Sugawara $U(1)$ contribution $-\frac{1}{2} n_{j}^{2}$ (and therefore, in view of the general arguments of Sect. 2.3, the first of the equations in (3.28) should hold irrespectively of decoupling equations).

Then, we arrive at a 'dressed' decoupling equation, which reads

$$
\left\{-\frac{1}{2 n_{i}^{2}} \frac{\partial^{2}}{\partial z_{i}^{2}}+\frac{1}{n_{i}} \sum_{j \neq i} \frac{1}{z_{j}-z_{i}}\left(n_{j} \frac{\partial}{\partial z_{i}}-n_{i} \frac{\partial}{\partial z_{j}}\right)\right\}\left\langle\Psi\left(z_{i}\right) \prod_{j \neq i} \Psi_{j}\left(z_{j}\right)\right\rangle=0
$$

with $\Psi$ being the dressed ' 21 ' operator. As we will see in Sect. 5.1, this equation is essentially the same as the Virasoro constraints (1.3).

\footnotetext{
${ }^{13}$ Dimensions refer to those as evaluated in the matter + 'Liouville' theory, by operator product expansions with the energy-momentum tensor $T(z)=\sum z^{-n-2} L_{n}$.

${ }^{14}$ This has the form of a 'continuum' version of the 'discrete' master equation from [17]; see ref. 21] for a discussion of this point. The same equation appeared recently in the solution of the Calogero model [51].
} 


\subsubsection{Level-3 reduction and the decoupling equation}

The story is much the same for level 3. The BRST invariance in our realization of the topological algebra serves as a principle that fixes the special form of a null state, which we will recognize below as being required for the application of the Kontsevich-Miwa transform. That is, for a level-3 topological algebra state

$$
\begin{aligned}
|\Xi\rangle= & \left(\alpha \mathcal{L}_{-1}^{3}-2 \mathcal{L}_{-2} \mathcal{L}_{-1}+\beta \mathcal{H}_{-3}+\gamma \mathcal{H}_{-2} \mathcal{L}_{-1}+\delta \mathcal{L}_{-3}+\epsilon \mathcal{H}_{-1} \mathcal{L}_{-2}\right. \\
& +\mu \mathcal{H}_{-1}^{2} \mathcal{L}_{-1}+\nu \mathcal{H}_{-1} \mathcal{L}_{-1}^{2}+\kappa \mathcal{H}_{-1} \mathcal{H}_{-2}+\rho \mathcal{H}_{-1}^{3} \\
& \left.+a \mathcal{Q}_{-2} \mathcal{G}_{-1}+e \mathcal{Q}_{-1} \mathcal{G}_{-2}+f \mathcal{L}_{-1} \mathcal{Q}_{-1} \mathcal{G}_{-1}+g \mathcal{H}_{-1} \mathcal{Q}_{-1} \mathcal{G}_{-1}\right)|\Phi\rangle
\end{aligned}
$$

we first demand that it be BRST-invariant, $\mathcal{Q}_{0}|\Xi\rangle=0$. It follows that $\rho=0$ and then $\beta=\kappa=0$ (no pure- $\mathcal{H}$ terms, as was the case for level 2) along with several other relations. Thus,

$$
\begin{aligned}
|\Xi\rangle= & \left(\alpha \mathcal{L}_{-1}^{3}-2 \mathcal{L}_{-2} \mathcal{L}_{-1}+\gamma \mathcal{H}_{-2} \mathcal{L}_{-1}+\delta \mathcal{L}_{-3}+2 b \mathcal{H}_{-1} \mathcal{L}_{-2}+g \mathcal{H}_{-1}^{2} \mathcal{L}_{-1}+2 f \mathcal{H}_{-1} \mathcal{L}_{-1}^{2}\right. \\
& \left.+a \mathcal{Q}_{-2} \mathcal{G}_{-1}+e \mathcal{Q}_{-1} \mathcal{G}_{-2}+f \mathcal{L}_{-1} \mathcal{Q}_{-1} \mathcal{G}_{-1}+g \mathcal{H}_{-1} \mathcal{Q}_{-1} \mathcal{G}_{-1}\right)|\Phi\rangle
\end{aligned}
$$

where $\gamma=2 a+2 f-g$. Further, from $\mathcal{H}_{1}|\Xi\rangle=0$ we find,

$$
\begin{aligned}
& f=\frac{3}{2 \mathrm{~h}}, \quad \alpha=\frac{3-\mathrm{c}}{3 \mathrm{~h}^{2}}, \quad a=\frac{\mathrm{c}+9-6 \mathrm{~h}}{6-2 \mathrm{c}}, \\
& e=\frac{-3 \mathrm{~h}}{3-\mathrm{c}}, \quad g=\frac{6}{3-\mathrm{c}}, \quad \gamma=\frac{\mathrm{c}+3-6 \mathrm{~h}}{3-\mathrm{c}}+\frac{3}{\mathrm{~h}}, \\
& \delta=-\gamma \mathrm{h}=\frac{6 \mathrm{~h}^{2}-\mathrm{h}(\mathrm{c}+3)-9+3 \mathrm{c}}{3-\mathrm{c}} .
\end{aligned}
$$

Then, the condition $\mathcal{G}_{1}|\Xi\rangle=0$ leads to the quadratic equation

$$
\mathrm{h}^{2}-\frac{\mathrm{c}+3}{3} \mathrm{~h}+\frac{2}{3}(\mathrm{c}-3)=0,
$$

whence

$$
h=\left\{\begin{array}{l}
\frac{c}{3}-1 \\
2
\end{array} .\right.
$$


Now, we use the same formula (3.21) as above to introduce a Miwa parameter $\hat{n}$ (this formula is independent of the level and reflects only the structure (3.7) of the topological current). Then it follows from $(3.32$

$$
\begin{array}{ll}
\alpha=\frac{1}{\hat{n}^{2}}, & \gamma=\left\{\begin{array}{l}
1-\frac{1}{\hat{n}^{2}} \\
-\frac{\hat{n}^{2}}{2}+\frac{1}{2},
\end{array}, \quad a=\left\{\begin{array}{l}
\frac{1}{2}+\frac{2}{\hat{n}^{2}} \\
-\frac{1}{2}
\end{array},\right.\right. \\
e=\left\{\begin{array}{l}
1 \\
-\frac{\hat{n}^{2}}{2},
\end{array}, \quad f=\left\{\begin{array}{l}
\frac{2}{\hat{n}^{2}} \\
\frac{3}{2}
\end{array}, \frac{3}{2 \hat{n}^{2}},\right.\right. \\
\delta=\hat{n}^{2}-1 .
\end{array}
$$

To see how the matter $\otimes U(1)$-null vector arises, consider now that part of (the first seven terms from) the RHS of (3.31) which contains no ghosts. It reads

$$
\begin{aligned}
\alpha \widehat{L}_{-1}^{3} & -2 \widehat{L}_{-2} \widehat{L}_{-1}+\gamma \sqrt{\frac{3-\mathrm{c}}{3}} I_{-2} \widehat{L}_{-1}+\delta \widehat{L}_{-3} \\
& +2 e \sqrt{\frac{3-\mathrm{c}}{3}} I_{-1} \widehat{L}_{-2}+g \frac{3-\mathrm{c}}{3} I_{-1}^{2} \widehat{L}_{-1}+2 f \sqrt{\frac{3-\mathrm{c}}{3}} I_{-1} \widehat{L}_{-1}^{2}
\end{aligned}
$$

We substitute here, as it follows from (3.34) and (3.7):

$$
\sqrt{\frac{3-\mathrm{c}}{3}}=\left\{\begin{array}{l}
-\hat{n} \\
\frac{2}{\hat{n}}
\end{array}\right.
$$

and act with the operator (3.36) onto the state $|\Psi\rangle$, where $|\Phi\rangle=|\Psi\rangle \otimes|0\rangle_{\mathrm{gh}}$. Then, for both the upper and the lower cases, we arrive at the following null state of the $(L, I)$ (or $(\widehat{L}, I)$ ) algebra:

$$
\begin{aligned}
|\Upsilon\rangle= & \left(\frac{1}{\hat{n}^{2}} \widehat{L}_{-1}^{3}-2 \widehat{L}_{-2} \widehat{L}_{-1}+\frac{1-\hat{n}^{2}}{\hat{n}} I_{-2} \widehat{L}_{-1}+\left(\hat{n}^{2}-1\right) \widehat{L}_{-3}-2 \hat{n} I_{-1} \widehat{L}_{-2}\right. \\
& \left.+2 I_{-1}^{2} \widehat{L}_{-1}+\frac{3}{\hat{n}} I_{-1} \widehat{L}_{-1}^{2}\right)|\Psi\rangle \\
= & \left(\frac{1}{\hat{n}^{2}} L_{-1}^{3}-2 L_{-2} L_{-1}+\frac{3-2 \hat{n}^{2}}{\hat{n}} I_{-2} L_{-1}+\left(\hat{n}^{2}-1\right) L_{-3}-2 \hat{n} I_{-1} L_{-2}\right. \\
& \left.+2 I_{-1}^{2} L_{-1}+\frac{3}{\hat{n}} I_{-1} L_{-1}^{2}+\frac{\left(\hat{n}^{2}-1\right)\left(\hat{n}^{2}-2\right)}{\hat{n}} I_{-3}+\left(2-\hat{n}^{2}\right) I_{-1} I_{-2}\right)|\Psi\rangle
\end{aligned}
$$

where, as follows from the previous formulae, 


$$
\hat{n}^{2}=\frac{13-d \pm \sqrt{(1-d)(25-d)}}{6}
$$

and

$$
Q=\frac{2}{\hat{n}}-\hat{n}
$$

so that the shift (3.3) takes the form

$$
\widehat{L}_{m}=L_{m}+\frac{1}{2}(m+1) \frac{2-\hat{n}^{2}}{\hat{n}} I_{m}
$$

The absence of the pure- $I$ terms in the null state obtained by the action of $\widehat{L}$ 's, eq. (3.38), is a consequence of the BRST invariance, which we have seen to suppress the pure- $\mathcal{H}$ terms. On the other hand it is just the condition that the pure- $I$ terms be absent that is crucial for the application of the Kontsevich-Miwa transform to the corresponding decoupling equation. This pattern repeats at higher levels, as the vanishing of the $I_{-1}^{l}$ term in the level- $l$ tensor product decoupling equation.

By the above construction, the 'dressed' decoupling equation corresponding to the above null vector will be just a particular case of implementing the BRST-invariance principle $\operatorname{Im} \mathcal{Q} \sim 0$ for correlators comprising only ghost-free representatives of chiral primary fields. The dimensionzero condition, as before, becomes the 'Kontsevich-Miwa' dressing prescription, which now reads

$$
\Delta_{j}=\frac{1}{2}\left(\hat{n}^{2}-2\right) \frac{n_{j}}{\hat{n}}
$$

Then, we arrive at the decoupling equation

$$
\widehat{\mathcal{O}}\left\langle\Psi\left(z_{i}\right) \prod_{j \neq i} \Psi_{j}\left(z_{j}\right)\right\rangle=0
$$

where 


$$
\begin{aligned}
& \widehat{\mathcal{O}}=\frac{1}{\hat{n}^{2}} \frac{\partial^{3}}{\partial z_{i}^{3}}+\sum_{j \neq i} \frac{1-\hat{n}^{2}}{\left(z_{j}-z_{i}\right)^{2}}\left(\frac{\partial}{\partial z_{j}}-\frac{n_{j}}{\hat{n}} \frac{\partial}{\partial z_{i}}\right) \\
& +\sum_{j \neq i} \frac{1}{z_{j}-z_{i}}\left(2 \frac{\partial^{2}}{\partial z_{j} \partial z_{i}}-3 \frac{n_{j}}{\hat{n}} \frac{\partial^{2}}{\partial z_{i}^{2}}\right)-2 \sum_{j \neq i k \neq i} \sum_{i} \frac{n_{k}}{\left(z_{j}-z_{i}\right)\left(z_{k}-z_{i}\right)}\left(\hat{n} \frac{\partial}{\partial z_{j}}-n_{j} \frac{\partial}{\partial z_{i}}\right) .
\end{aligned}
$$

This decoupling operator is further studied in Sect. 5, where we show how it can be related to the Virasoro constraints in terms of the KP times $t_{r}$.

\section{Appendix: DDK formalism from $N=2$}

As has been noticed in the Introduction, our starting point, the topological algebra, allows us to recover, besides the 'Kontsevich-Miwa'-dressed matter, also the DDK dressing prescription. To this end, one should consider, instead of the ansatz (3.6), (3.7), (3.10), (3.11), another reduction of the topological algebra to matter + 'Liouville' + ghosts [40. Namely, let us split away from the topological generators a spin-2 ghost system. Using the same notations as in Sect. 3 (although the ghost system is actually different), we write

$$
\mathcal{L}_{m}=\widehat{L}_{m}+l_{m}, \quad l_{m} \equiv \sum_{n \in \mathbf{Z}}(m+n): b_{m-n} c_{n}:
$$

Then the central charge read off from the $\left[\widehat{L}_{m}, \widehat{L}_{n}\right]$ commutator is 26 . Further, the DDK dressing prescription 31, 32 can be recovered as follows. Recall that for a spin- $\lambda$ ghost system, the $S L_{2}$ invariant vacuum state $|0\rangle$ is characterized by [41,

$$
b_{>-\lambda}|0\rangle=c_{\geq \lambda}|0\rangle=0
$$

[a particular case of which we have already met in (3.1)]. For the reparametrization ghosts it is thus only the $c_{\geq 2}$ out of the $c_{n}$ modes that annihilate the vacuum, which allows us to split the topological algebra states as

$$
|\Phi\rangle=|\Psi\rangle \otimes c_{1}|0\rangle
$$

In view of (3.A1) and (3.17), this implies that the $\widehat{L}$-dimension of $\Psi$ is $\widehat{\Delta}=1$. Clearly, the same holds for all the other chiral primary states $\Phi_{j}$ : splitting them as 


$$
\left|\Phi_{j}\right\rangle=\left|\Psi_{j}\right\rangle \otimes c_{1}|0\rangle
$$

we arrive at the conditions

$$
\widehat{\Delta}_{j}=1
$$

which will fix the DDK dressing prescription as soon as the background charges are known. Thus, to complete the derivation, let us first give the expression for the BRST current modes:

$$
\mathcal{Q}_{m}=2 \sum_{p \in \mathbf{Z}} c_{m-p} \widehat{L}_{p}+\sum_{p, r \in \mathbf{Z}}(p-r): b_{m-p-r} c_{p} c_{r}:+2 \sqrt{\frac{3-c}{3}} m \sum_{p \in \mathbf{Z}} c_{m-p} I_{p}+\frac{c}{3}\left(m^{2}-m\right) c_{m}
$$

As to the $\widehat{L}_{p}$ generators, note that the comment below eq. (3.11) applies in the present case as well. Now, with the superpartner of the energy-momentum tensor being

$$
\mathcal{G}_{m}=b_{m},
$$

we use the $\left\{\mathcal{G}_{m}, \mathcal{Q}_{n}\right\}$ commutator to find that the topological current is given by

$$
\mathcal{H}_{m}=\sum_{n \in \mathbf{Z}}: b_{m-n} c_{n}:-\sqrt{\frac{3-\mathrm{c}}{3}} I_{m} .
$$

From the $\left[\mathcal{L}_{m}, \mathcal{H}_{n}\right]$ commutator we can now derive

$$
\left[\widehat{L}_{m}, I_{n}\right]=-n I_{m+n}-\sqrt{\frac{3}{3-\mathrm{c}}} \frac{\mathrm{c}-9}{6}\left(m^{2}+m\right) \delta_{m+n, 0} .
$$

The anomaly thus emerging can be expressed as

$$
-\sqrt{\frac{3}{3-c}} \frac{\mathrm{c}-9}{6}=-\frac{1}{2} \sqrt{\frac{25-d}{3}} \equiv-\frac{1}{2} Q_{\mathrm{L}},
$$

where $Q_{\mathrm{L}}$ is the standard background charge of the Liouville scalar. Let us note that the construction (3.A1), (3.A6)-(3.A8), [40], for the topological generators was generalized recently to include the $\mathrm{W}_{3}$ generators [53]. 
The existence of just two 'bosonizations' of the topological algebra in terms of matter, 'Liouville' and ghosts, follows, according to an observation by Dijkgraaf [52], from the two possible twistings of the proper $N=2$ algebra. Both the 'spin-1' construction of Sect. 3.1 and the above 'spin-2' version are different twistings of a 'spin- $\frac{3}{2}$ ' construction. Indeed, consider the $N=2$ algebra (we have once again changed the notations, the $\mathcal{L}_{p}$ now being the untwisted generators)

$$
\begin{aligned}
{\left[\mathcal{L}_{m}, \mathcal{L}_{n}\right] } & =(m-n) \mathcal{L}_{m+n}+\frac{\mathrm{c}}{12}\left(m^{3}-m\right) \delta_{m+n, 0}, & {\left[\mathcal{H}_{m}, \mathcal{H}_{n}\right]=\frac{\mathrm{c}}{3} m \delta_{m+n, 0}, } \\
{\left[\mathcal{L}_{m}, \mathcal{G}_{r}^{ \pm}\right] } & =\left(\frac{m}{2}-r\right) \mathcal{G}_{m+r}^{ \pm}, & {\left[\mathcal{H}_{m}, \mathcal{G}_{r}^{ \pm}\right]= \pm \mathcal{G}_{m+r}^{ \pm}, } \\
{\left[\mathcal{L}_{m}, \mathcal{H}_{n}\right] } & =-n \mathcal{H}_{m+n} & \\
\left\{\mathcal{G}_{r}^{-}, \mathcal{G}_{s}^{+}\right\} & =2 \mathcal{L}_{r+s}-(r-s) \mathcal{H}_{r+s}+\frac{\mathrm{c}}{3}\left(r^{2}-\frac{1}{4}\right) \delta_{r+s, 0}, &
\end{aligned}
$$

where the $\mathcal{G}^{ \pm}$have spin $\frac{3}{2}$ and are half-integer moded. One of the fields $\mathcal{G}^{ \pm}(z)$ can be identified with the $b$ field of a spin- $\frac{3}{2} b c$ system:

$$
b_{r}=\mathcal{G}_{r}^{-} .
$$

Let then $c$ be the conjugate, spin- $\left(-\frac{1}{2}\right)$ field. The $b c$ theory is defined as before, by $\left\{b_{r}, c_{s}\right\}=$ $\delta_{r+s, 0}$ and relations (3.A2) for $\lambda=\frac{3}{2}$; the energy-momentum tensor and the ghost current read

$$
l_{m}=\sum_{r}\left(r+\frac{m}{2}\right): b_{m-r} c_{r}:, \quad i_{m}=-\sum_{r}: b_{m-r} c_{r}:
$$

Now, let

$$
Q=-\frac{\mathrm{c}+3}{\sqrt{3(3-\mathrm{c})}}, \quad Q_{\mathrm{L}}=\frac{\mathrm{c}-9}{\sqrt{3(3-\mathrm{c})}}
$$

(which is real for $\mathrm{c}<3$ ), and represent the $U(1)$ current $\mathcal{H}$ as

$$
\mathcal{H}_{m}=i_{m}-\frac{1}{2}\left(Q_{\mathrm{L}}-Q\right) I_{m}
$$


where, as before, $\left[I_{m}, I_{n}\right]=-m \delta_{m+n, 0}$. Similarly, for the Virasoro generators $L_{m}$, introduced via

$$
\mathcal{L}_{m}=L_{m}+\frac{1}{4}\left(Q_{\mathrm{L}}+Q\right)(m+1) I_{m}+l_{m}
$$

we find the central charge $d+1$ with $d$ given by (1.9), and therefore, after subtracting the Sugawara $U(1)$ contribution as in (3.12), we arrive at the 'matter' Virasoro generators with central charge $d$. We have already seen that, when reexpressed in terms of $d, Q_{\mathrm{L}}$ becomes the standard Liouville background charge $\sqrt{\frac{25-d}{3}}$, while $Q$ coincides with the matter background charge $\sqrt{\frac{1-d}{3}}$. Finally, we construct

$\mathcal{G}_{r}^{+}=2 \sum_{n} c_{r-n} L_{n}+\sum_{s, q}(s-q): b_{r-s-q} c_{s} c_{q}:+\sum_{n}\left(Q_{\mathrm{L}} n+\left(Q-Q_{\mathrm{L}}\right) r+\frac{1}{2} Q+\frac{1}{2} Q_{\mathrm{L}}\right) c_{r-n} I_{n}+\frac{\mathrm{C}}{3}\left(r^{2}-\frac{1}{4}\right) c_{r}$.

There are, as usual, just two possibilities to twist the algebra (3.A11), with either $\mathcal{G}^{+}$or $\mathcal{G}^{-}$ acquiring spin 2. The first twisting is accomplished by setting

$$
\begin{array}{rlrl}
\mathcal{L}_{m}^{(1)} & =\mathcal{L}_{m}+\frac{1}{2}(m+1) \mathcal{H}_{m}, & \\
c_{m}^{(1)} & =c_{m+\frac{1}{2}}, & b_{m}^{(1)} & =b_{m-\frac{1}{2}} \\
\mathcal{H}_{m}^{(1)} & =\mathcal{H}_{m}, & \\
\mathcal{G}_{m}^{(1)} & =\mathcal{G}_{m+\frac{1}{2}}^{+}, & \mathcal{Q}_{n}^{(1)}=\mathcal{G}_{n-\frac{1}{2}}^{-},
\end{array}
$$

which, after dropping the superscript ${ }^{(1)}$, reproduces our spin-1 construction in Sect. 3.1, with

$$
\widehat{L}_{m}=L_{m}+\frac{1}{2} Q(m+1) I_{m}
$$

Alternatively, to make $\mathcal{G}^{-}$of spin 2 , we set

$$
\begin{aligned}
\mathcal{L}_{m}^{(2)} & =\mathcal{L}_{m}-\frac{1}{2}(m+1) \mathcal{H}_{m}, \\
c_{m}^{(2)} & =c_{m-\frac{1}{2}}, \quad b_{m}^{(2)}=b_{m+\frac{1}{2}}, \\
\mathcal{H}_{m}^{(2)} & =-\mathcal{H}_{m}, \\
\mathcal{G}_{m}^{(2)} & =\mathcal{G}_{m+\frac{1}{2}}^{-}, \quad \mathcal{Q}_{n}^{(2)}=\mathcal{G}_{n-\frac{1}{2}}^{+}, \\
\widehat{L}_{m} & =L_{m}+\frac{1}{2} Q_{\mathrm{L}}(m+1) I_{m},
\end{aligned}
$$


which leads us back to the above spin-2 construction.

The rôles of the BRST operator $\mathcal{Q}_{0}$ and its spin-2 superpartner $\mathcal{G}_{0}$ are therefore 'dual' to each other in the two twisted constructions, with one of these operators being 'simple' (coinciding with the $b$ field) and the other one 'complicated'. To preserve the physical content (the cohomologies), one should therefore consider equivariant cohomologies, thus introducing both $\mathcal{Q}_{0}$ and $\mathcal{G}_{0}$ into the consideration, so as to ensure the equivalence between the two twisted versions.

\section{Dressed null states via a direct construction}

On the way from the twisted $N=2$ symmetry to the Virasoro constraints imposed by the operators (1.1), we have arrived at the 'dressed decoupling equations', such as (3.29) and (3.43) (3.44). These can also be obtained by a direct construction, which may be useful in practice. The construction amounts to specifying a way to restrict from the most general tensor product decoupling equation in the matter $\otimes$ 'Liouville' theory. As was noted above, a characteristic feature that the null states (3.25) and (3.38) inherit from the BRST invariance, is the absence of the a priori possible $I_{-1}^{l}$ terms where $l$ is the level. This property, as we will see, can be used to characterize the required null states, and is one out of the two basic conditions that we impose, the other one being the 'Kontsevich-Miwa' condition on dressing field operators. Together, these two will bring the corresponding decoupling operators to the final form amenable to the Kontsevich-Miwa transform, which at the same time coincides with what would follow from the $N=2$ machinery. We first demonstrate this approach for level 3, and then use it to arrive at the appropriately dressed decoupling equation at level 4. The reader wishing to perform the next step, the actual transformation to the Virasoro constraints, may skip to Sect. 5 .

In the tensor product theory comprising the energy-momentum tensor $T(z)=\sum_{n \in \mathbf{Z}} L_{n} z^{-n-2}$ and the $U(1)$ current $I(z)=\sum_{n \in \mathbf{Z}} I_{n} z^{-n-1}$, with the commutation relations as written out in (3.2), let $\Psi$ be a primary field with conformal dimension $\Delta$ and $U(1)$ charge $\hat{n}$. We now consider levels 3 and 4 separately. 


\subsection{Dressing at level 3}

It is straightforward to check that the most general level-3 null vector w.r.t. the semidirect product of Virasoro with the $U(1)$ will result from the action on $|\Psi\rangle$ of the following operator:

$$
\begin{aligned}
\mathcal{O} \equiv & \delta L_{-3}-2 L_{-2} L_{-1}+\frac{1}{\delta+1} L_{-1}^{3}-\frac{\delta+1-3 \hat{n}^{2}}{\delta+1} I_{-1}^{2} L_{-1}-\hat{n} \frac{2 \delta-1}{\delta+1} I_{-2} L_{-1}-2 \hat{n} I_{-1} L_{-2} \\
& +\frac{3 \hat{n}}{\delta+1} I_{-1} L_{-1}^{2}+\frac{\delta^{2}+\delta-2 \delta \hat{n}^{2}+\hat{n}^{2}}{\delta+1} I_{-1} I_{-2}-\hat{n} \frac{\delta+1-\hat{n}^{2}}{\delta+1} I_{-1}^{3}+\hat{n} \frac{\delta(\delta-1)}{\delta+1} I_{-3}
\end{aligned}
$$

provided $\delta \equiv \delta_{i}$, which is the matter dimension of $\Psi$, according to

$$
\delta=\Delta-\left(-\frac{\hat{n}^{2}}{2}\right)
$$

is given by

$$
\delta=\frac{7-d \mp \sqrt{(1-d)(25-d)}}{6} .
$$

(It is understood that for the $\left(p^{\prime}, p\right)$ minimal model, $d=1-\frac{6\left(p^{\prime}-p\right)^{2}}{p^{\prime} p}$.)

As explained above, we can convert the condition $\mathcal{O}|\Psi\rangle=0$ into a decoupling equation of the form of (3.43), in which this time we have

$$
\begin{aligned}
\widehat{\mathcal{O}} \equiv & \frac{1}{\delta+1} \frac{\partial^{3}}{\partial z_{i}^{3}}-\delta \sum_{j \neq i}\left\{\frac{1}{\left(z_{j}-z_{i}\right)^{2}} \frac{\partial}{\partial z_{j}}-\frac{2 \Delta_{j}}{\left(z_{j}-z_{i}\right)^{3}}\right\}+2 \sum_{j \neq i}\left\{\frac{1}{z_{j}-z_{i}} \frac{\partial}{\partial z_{j}}-\frac{\Delta_{j}}{\left(z_{j}-z_{i}\right)^{2}}\right\} \frac{\partial}{\partial z_{i}} \\
& -\frac{\delta-3 \hat{n}^{2}+1}{\delta+1} \sum_{\substack{j, k \\
j \neq i, k \neq i}} \frac{n_{j} n_{k}}{\left(z_{j}-z_{i}\right)\left(z_{k}-z_{i}\right)} \frac{\partial}{\partial z_{i}}+\hat{n} \frac{2 \delta-1}{\delta+1} \sum_{j \neq i} \frac{n_{j}}{\left(z_{j}-z_{i}\right)^{2}} \frac{\partial}{\partial z_{i}} \\
& -2 \hat{n} \sum_{\substack{j, k \\
j \neq i, k \neq i}} \frac{n_{j}}{\left(z_{j}-z_{i}\right)\left(z_{k}-z_{i}\right)} \frac{\partial}{\partial z_{k}}-\frac{3 \hat{n}}{\delta+1} \sum_{j \neq i} \frac{n_{j}}{z_{j}-z_{i}} \frac{\partial^{2}}{\partial z_{i}^{2}} \\
& +\frac{\delta^{2}+\delta-2 \hat{n}^{2} \delta+\hat{n}^{2}}{\delta+1} \sum_{\substack{j, k \\
j \neq i, k \neq i}} \frac{n_{j} n_{k}}{\left(z_{j}-z_{i}\right)\left(z_{k}-z_{i}\right)^{2}}+2 \hat{n} \sum_{\substack{j, k \\
j \neq i, k \neq i}} \frac{n_{j} \Delta_{k}}{\left(z_{j}-z_{i}\right)\left(z_{k}-z_{i}\right)^{2}} \\
& +\hat{n} \frac{\delta+1-\hat{n}^{2}}{\delta+1} \sum_{\substack{j, k, l \\
j \neq i, k \neq i, l \neq i}} \frac{n_{j} n_{k} n_{l}}{\left(z_{j}-z_{i}\right)\left(z_{k}-z_{i}\right)\left(z_{l}-z_{i}\right)}-\hat{n} \frac{\delta(\delta-1)}{\delta+1} \sum_{j \neq i} \frac{n_{j}}{\left(z_{j}-z_{i}\right)^{3}} .
\end{aligned}
$$


However, the null vector $\mathcal{O}|\Psi\rangle$ with $\mathcal{O}$ as in (4.1) allows too much arbitrariness, as the values of $\Delta$ and $\hat{n}^{2}$ cannot be fixed separately: there is a one-parametric freedom which reflects the fact that we have extended the matter theory by a current. This extra freedom can be killed by restricting to a more special form of the operator (4.1), achieved by demanding that the coefficient in front of the $I_{-1}^{3}$ term vanish, which amounts to setting

$$
\delta=\hat{n}^{2}-1
$$

The next step consists in restricting to a subsector of those operators whose dimensions and $U(1)$ charges satisfy the 'Kontsevich-Miwa' dressing condition (3.42). This allows us, in the decoupling equation, to get rid of the terms

$$
\sum_{\substack{j, k \\ j \neq i, k \neq i}} \frac{\hat{n}_{j}}{\left(z_{i}-z_{j}\right)\left(z_{k}-z_{i}\right)^{2}}\left\{\left(2-\hat{n}^{2}\right) n_{k}+2 \hat{n} \Delta_{k}\right\}\left\langle\Psi\left(z_{i}\right) \prod_{j \neq i} \Psi_{j}\left(z_{j}\right)\right\rangle,
$$

which do not have analogues in (3.43), (3.44). As a result of (4.5) and (3.42), the decoupling operator (4.4) takes exactly the form (3.44). The present derivation allows us to see by what kind of restrictions this special form is characterized among the general family (4.4). In the next subsection we demonstrate how a similar argument allows us to arrive at the dressed null vectors at level 4 .

\subsection{Dressing at level 4}

At level 4, the null vectors exist over ' 41 ' (and '14') and '22' states, and so do of course the dressed null vectors. We are going to consider them separately.

\subsubsection{Dressed null vectors over the '41' state}

Consider first the '41' case. The general null vector in the semidirect product of Virasoro with $U(1)$ reads 


$$
\begin{aligned}
&\left|\Upsilon_{41}\right\rangle=\left(25\left(2 \Delta+\hat{n}^{2}+3\right) L_{-2} L_{-1}^{2}+50\left(2 \Delta+\hat{n}^{2}+3\right) \hat{n} I_{-1} L_{-2} L_{-1}\right. \\
&-\left(8 \hat{n}^{4}+32 \hat{n}^{2} \Delta+23 \hat{n}^{2}+32 \Delta^{2}+46 \Delta-3\right) L_{-3} L_{-1}-75 \hat{n} I_{-1} L_{-1}^{3} \\
&-\frac{75}{4} L_{-1}^{4}+25\left(-2 \hat{n}^{3}+2 \hat{n} \Delta+3 \hat{n}\right) I_{-1}^{3} L_{-1}+25\left(\frac{3}{2}-4 \hat{n}^{2}+\Delta\right) I_{-1}^{2} L_{-1}^{2} \\
&+25\left(\hat{n}^{3}+2 \hat{n} \Delta-\frac{3}{2} \hat{n}\right) I_{-2} L_{-1}^{2}-\left(27+3 \hat{n}^{4}+12 \hat{n}^{2} \Delta+18 \hat{n}^{2}+12 \Delta^{2}+36 \Delta\right) L_{-2}^{2} \\
&+\left(\frac{81}{5}+\frac{8}{5} \hat{n}^{6}+\frac{48}{5} \hat{n}^{4} \Delta+\frac{32}{5} \hat{n}^{4}+\frac{96}{5} \hat{n}^{2} \Delta^{2}+\frac{128}{5} \hat{n}^{2} \Delta+\frac{51}{5} \hat{n}^{2}+\frac{64}{5} \Delta^{3}+\frac{128}{5} \Delta^{2}+\frac{102}{5} \Delta\right) L_{-4} \\
&+\left(-8 \hat{n}^{5}-32 \hat{n}^{3} \Delta+27 \hat{n}^{3}-32 \hat{n} \Delta^{2}+54 \hat{n} \Delta+3 \hat{n}\right) I_{-3} L_{-1} \\
&+\left(-27+22 \hat{n}^{4}+38 \hat{n}^{2} \Delta+57 \hat{n}^{2}-12 \Delta^{2}-36 \Delta\right) I_{-1}^{2} L_{-2} \\
&-\left(6 \hat{n}^{5}+24 \hat{n}^{3} \Delta+11 \hat{n}^{3}+24 \hat{n} \Delta^{2}+22 \hat{n} \Delta-21 \hat{n}\right) I_{-2} L_{-2} \\
&-\left(8 \hat{n}^{5}+32 \hat{n}^{3} \Delta+23 \hat{n}^{3}+32 \hat{n} \Delta^{2}+46 \hat{n} \Delta-3 \hat{n}\right) I_{-1} L_{-3} \\
&+\left(3+42 \hat{n}^{4}+68 \hat{n}^{2} \Delta-98 \hat{n}^{2}-32 \Delta^{2}-46 \Delta\right) I_{-2} I_{-1} L_{-1} \\
&+\left(-\frac{27}{4}-7 \hat{n}^{4}+22 \hat{n}^{2} \Delta+33 \hat{n}^{2}-3 \Delta^{2}-9 \Delta\right) I_{-1}^{4} \\
&+\left(\frac{8}{5} \hat{n}^{7}+\right.\left.\frac{48}{5} \hat{n}^{5} \Delta-\frac{38}{5} \hat{n}^{5}+\frac{96}{5} \hat{n}^{3} \Delta^{2}-\frac{152}{5} \hat{n}^{3} \Delta+\frac{6}{5} \hat{n}^{3}+\frac{64}{5} \hat{n} \Delta^{3}-\frac{152}{5} \hat{n} \Delta^{2}+\frac{12}{5} \hat{n} \Delta+\frac{27}{10} \hat{n}\right) I_{-4} \\
&+\left(\frac{81}{10}-\frac{11}{5} \hat{n}^{6}-\frac{36}{5} \hat{n}^{4} \Delta+\frac{51}{5} \hat{n}^{4}-\frac{12}{5} \hat{n}^{2} \Delta^{2}+\frac{134}{5} \hat{n}^{2} \Delta-\frac{63}{20} \hat{n}^{2}+\frac{32}{5} \Delta^{3}+\frac{64}{5} \Delta^{2}+\frac{51}{5} \Delta\right) I_{-2}^{2} \\
&+\left(-\frac{54}{5}-\right.\left.\frac{32}{5} \hat{n}^{6}-\frac{112}{5} \hat{n}^{4} \Delta+\frac{152}{5} \hat{n}^{4}-\frac{64}{5} \hat{n}^{2} \Delta^{2}+\frac{338}{5} \hat{n}^{2} \Delta-\frac{24}{5} \hat{n}^{2}+\frac{64}{5} \Delta^{3}+\frac{68}{5} \Delta^{2}-\frac{78}{5} \Delta\right) I_{-3} I_{-1} \\
&\left.+\left(14 \hat{n}^{5}+6 \hat{n}^{3} \Delta-66 \hat{n}^{3}-44 \hat{n} \Delta^{2}-57 \hat{n} \Delta+\frac{27}{2} \hat{n}\right) I_{-2} I_{-1}^{2}\right)\left|\Psi_{41}\right\rangle .
\end{aligned}
$$

Here, as before, the 'dressed' dimension $\Delta$ is related to the matter dimension $\delta$ of $\Psi$ via eq. (4.2), while

$$
\delta=\frac{41-5 d+5 \sqrt{(1-d)(25-d)}}{16} .
$$

Now, there are two possibilities to get rid of the $I_{-1}^{4}$ term. One of these is to set (cf. (4.5))

$$
\hat{n}^{2}=\frac{6 \Delta+9}{2}=\frac{6 \delta+9}{5} .
$$

Then, eq. (4.7) becomes 


$$
\begin{aligned}
\left|\Upsilon_{41}\right\rangle= & \left(L_{-2} L_{-1}^{2}+2 \hat{n} I_{-1} L_{-2} L_{-1}-\left(\frac{8 \hat{n}^{2}}{15}-1\right) L_{-3} L_{-1}-\frac{9}{5 \hat{n}} I_{-1} L_{-1}^{3}-\frac{9}{20 \hat{n}^{2}} L_{-1}^{4}\right. \\
& -\frac{4 \hat{n}}{5} I_{-1}^{3} L_{-1}-\frac{11}{5} I_{-1}^{2} L_{-1}^{2}+\left(\hat{n}-\frac{27}{10 \hat{n}}\right) I_{-2} L_{-1}^{2}-\frac{\hat{n}^{2}}{5} L_{-2}^{2} \\
& +\left(\frac{8 \hat{n}^{4}}{45}-\frac{8 \hat{n}^{2}}{15}+\frac{3}{5}\right) L_{-4}-\left(\frac{8 \hat{n}^{3}}{15}-3 \hat{n}+\frac{18}{5 \hat{n}}\right) I_{-3} L_{-1}+\frac{4}{5} \hat{n}^{2} I_{-1}^{2} L_{-2} \\
& -\left(\frac{2 \hat{n}^{3}}{5}-\hat{n}\right) I_{-2} L_{-2}-\left(\frac{8 \hat{n}^{3}}{15}-\hat{n}\right) I_{-1} L_{-3}+\left(\frac{22 \hat{n}^{2}}{15}-\frac{22}{5}\right) I_{-2} I_{-1} L_{-1} \\
& +\frac{3}{5 \hat{n}}\left(\frac{8 \hat{n}^{6}}{27}-\frac{22 \hat{n}^{4}}{9}+6 \hat{n}^{2}-\frac{9}{2}\right) I_{-4}-\frac{3}{5}\left(\frac{5 \hat{n}^{4}}{27}-\frac{11 \hat{n}^{2}}{9}-\frac{33}{4}\right) I_{-2}^{2} \\
& \left.-\frac{3}{5}\left(\frac{16 \hat{n}^{4}}{27}-\frac{34 \hat{n}^{2}}{9}-27\right) I_{-3} I_{-1}+\left(\frac{4 \hat{n}^{3}}{15}-\frac{6 \hat{n}}{5}\right) I_{-2} I_{-1}^{2}\right)\left|\Psi_{41}\right\rangle .
\end{aligned}
$$

The corresponding decoupling operator $\widehat{\mathcal{O}} \equiv \widehat{\mathcal{O}}^{(4)}[$ see $(3.43)]$ takes the form

$$
\begin{aligned}
\widehat{\mathcal{O}}^{(4)}= & -\frac{9}{20 n_{i}^{2}} \partial_{i}^{4}+\sum_{j \neq i} \frac{1}{z_{j}-z_{i}}\left(-\partial_{j} \partial_{i}^{2}+\frac{9 n_{j}}{5 n_{i}} \partial_{i}^{3}\right) \\
& +\sum_{j \neq i} \frac{1}{\left(z_{j}-z_{i}\right)^{2}}\left\{\left(\frac{8 n_{i}^{2}}{15}-1\right) \partial_{i} \partial_{j}-\left(\frac{2 n_{i}}{3}-\frac{6}{5 n_{i}}\right) n_{j} \partial_{i}^{2}\right\} \\
& +\sum_{j \neq i} \sum_{k \neq i} \frac{1}{\left(z_{j}-z_{i}\right)\left(z_{k}-z_{i}\right)}\left(2 n_{i} n_{k} \partial_{j} \partial_{i}-\frac{11}{5} n_{j} n_{k} \partial_{i}^{2}-\frac{n_{i}^{2}}{5} \partial_{k} \partial_{j}\right) \\
& +\sum_{j \neq i} \frac{1}{\left(z_{j}-z_{i}\right)^{3}}\left(-\frac{8 n_{i}^{3}}{45}+\frac{11 n_{i}}{15}-\frac{3}{5 n_{i}}\right)\left(n_{i} \partial_{j}-n_{j} \partial_{i}\right) \\
& +\sum_{j \neq i} \sum_{k \neq i} \frac{1}{\left(z_{j}-z_{i}\right)\left(z_{k}-z_{i}\right)^{2}}\left\{\left(1-\frac{8 n_{i}^{2}}{15}\right) n_{j}\left(n_{i} \partial_{k}-n_{k} \partial_{i}\right)+\left(\frac{2}{5}-\frac{4 n_{i}^{2}}{15}\right) n_{k}\left(n_{i} \partial_{j}-n_{j} \partial_{i}\right)\right\} \\
& -\frac{4}{5} \sum_{j \neq i} \sum_{i k \neq i l \neq i} \frac{n_{i} n_{k} n_{l}}{\left(z_{j}-z_{i}\right)\left(z_{k}-z_{i}\right)\left(z_{l}-z_{i}\right)}\left(n_{i} \partial_{j}-n_{j} \partial_{i}\right),
\end{aligned}
$$

where $\partial_{j}=\partial / \partial z_{j}$ (and where, let us recall, $n_{i} \equiv \hat{n}$ ).

We have seen that the level-2 and level-3 dressing conditions (3.28) and (3.42) are described by the same 'universal' (level-independent) formula

$$
\Delta_{j}=-\frac{1}{2} Q n_{j},
$$


where $Q$ is the corresponding matter background charge, so that the ${ }^{-}$-dimensions are always zero,

$$
\widehat{\Delta}_{j}=0
$$

which is what remains of the underlying topological symmetry (zero 'twisted' dimensions of the chiral primary fields). The same condition (4.12) holds in the present case as well, with the background charge, as deduced from (4.8) and (4.9), being given in terms of the Miwa parameter $\hat{n} \equiv n_{i}$ as

$$
Q=\frac{3}{\hat{n}}-\frac{2 \hat{n}}{3} \quad(l=4)
$$

In the next section we will show that this is indeed the background charge of the Virasoro generators of the type of (11.1), through which the operator $\widehat{\mathcal{O}}^{(4)}$ factorizes.

The other possibility to kill the $I_{-1}^{4}$ term over the ' 41 ' state is

$$
\hat{n}^{2}=\frac{2 \Delta+3}{14}=\frac{2 \delta+3}{15}
$$

with $\delta$ as in (4.8). The relation between the Miwa parameter and the background charge is now

$$
Q=2 \hat{n}-\frac{1}{\hat{n}}
$$

However, with this value of $Q$ the dressing condition (4.12) would not be satisfied unless $\hat{n}^{2}=\frac{1}{4}$. The matter central charge is then $d=-2$, while the corresponding null vector itself is in the intersection of the ' 22 ' and ' 41 ' null vectors, which we discuss later, at the end of Sect. 4.2.2.

\subsubsection{Dressed null vectors over the '22' state}

As has been noted above, there are more possibilities at level 4: in addition to (4.7), a null vector exists over the ' 22 ' state: 


$$
\begin{aligned}
\left|\Upsilon_{22}\right\rangle= & \left(\left(2 \Delta+\hat{n}^{2}+3\right) L_{-2} L_{-1}^{2}+2 \hat{n}\left(2 \Delta+\hat{n}^{2}+3\right) I_{-1} L_{-2} L_{-1}+\left(\hat{n}^{2}+2 \Delta-\frac{3}{2}\right) L_{-3} L_{-1}\right. \\
& -3 \hat{n} I_{-1} L_{-1}^{3}-\frac{3}{4} L_{-1}^{4}+\left(-2 \hat{n}^{3}+2 \hat{n} \Delta+3 \hat{n}\right) I_{-1}^{3} L_{-1}+\left(\frac{3}{2}-4 \hat{n}^{2}+\Delta\right) I_{-1}^{2} L_{-1}^{2} \\
& +\left(\hat{n}^{3}+2 \hat{n} \Delta-\frac{3}{2} \hat{n}\right) I_{-2} L_{-1}^{2}-\left(\frac{1}{3} \hat{n}^{4}+\frac{4}{3} \hat{n}^{2} \Delta+2 \hat{n}^{2}+\frac{4}{3} \Delta^{2}+4 \Delta\right) L_{-2}^{2}+\left(\frac{3}{2} \hat{n}^{2}+3 \Delta\right) L_{-4} \\
& +\left(3 \hat{n}^{3}+6 \hat{n} \Delta-\frac{3}{2} \hat{n}\right) I_{-3} L_{-1}+\left(\frac{2}{3} \hat{n}^{4}+\frac{2}{3} \hat{n}^{2} \Delta+\hat{n}^{2}-\frac{4}{3} \Delta^{2}-4 \Delta\right) I_{-1}^{2} L_{-2} \\
& -\left(\frac{2}{3} \hat{n}^{5}+\frac{8}{3} \hat{n}^{3} \Delta+3 \hat{n}^{3}+\frac{8}{3} \hat{n} \Delta^{2}+6 \hat{n} \Delta-3 \hat{n}\right) I_{-2} L_{-2} \\
& +\left(\hat{n}^{3}+2 \hat{n} \Delta-\frac{3}{2} \hat{n}\right) I_{-1} L_{-3}+\left(-\frac{3}{2}+2 \hat{n}^{4}+4 \hat{n}^{2} \Delta-2 \hat{n}^{2}+2 \Delta\right) I_{-2} I_{-1} L_{-1} \\
& +\left(-\frac{1}{3} \hat{n}^{4}+\frac{2}{3} \hat{n}^{2} \Delta+\hat{n}^{2}-\frac{1}{3} \Delta^{2}-\Delta\right) I_{-1}^{4}+\left(-\frac{2}{3} \hat{n}^{5}-\frac{8}{3} \hat{n}^{3} \Delta+\frac{1}{2} \hat{n}^{3}-\frac{8}{3} \hat{n} \Delta^{2}+\hat{n} \Delta\right) I_{-4} \\
& +\left(-\frac{1}{3} \hat{n}^{6}-\frac{4}{3} \hat{n}^{4} \Delta-\hat{n}^{4}-\frac{4}{3} \hat{n}^{2} \Delta^{2}-2 \hat{n}^{2} \Delta+\frac{3}{2} \hat{n}^{2}+\frac{3}{2} \Delta\right) I_{-2}^{2} \\
+\left(\frac{8}{3} \hat{n}^{4}\right. & \left.\left.+\frac{14}{3} \hat{n}^{2} \Delta-2 \hat{n}^{2}-\frac{4}{3} \Delta^{2}-\Delta\right) I_{-3} I_{-1}+\left(\frac{2}{3} \hat{n}^{5}+\frac{2}{3} \hat{n}^{3} \Delta-2 \hat{n}^{3}-\frac{4}{3} \hat{n} \Delta^{2}-\hat{n} \Delta\right) I_{-2} I_{-1}^{2}\right)\left|\Psi_{22}\right\rangle
\end{aligned}
$$

As before, we demand that the $I_{-1}^{4}$ term vanish. The corresponding equation reads, in terms of the matter dimension $\delta$ [see (4.2)],

$$
9 \hat{n}^{4}-12 \hat{n}^{2} \delta-18 \hat{n}^{2}+4 \delta^{2}+12 \delta=0
$$

whence

$$
\hat{n}^{2}=\frac{2 \delta+3 \pm 3}{3}
$$

If we recall that $\delta$ is now related to the matter central charge $d$ via $\delta=\frac{1-d}{8}$, we get

$$
\hat{n}^{2}=\frac{13-d \pm 12}{12}
$$

To see what these two possibilities mean, consider the coefficient $C_{j}$ of the terms

$$
\sum_{j \neq i} \sum_{k \neq i} \frac{C_{j} \hat{n}_{k}}{\left(z_{j}-z_{i}\right)^{3}\left(z_{k}-z_{i}\right)}
$$

in the decoupling equation corresponding to the null vector (4.17). It is equal to

$$
C_{j}=-2 \hat{n}\left(\hat{n}^{2}+2 \Delta-\frac{3}{2}\right) \Delta_{j}+\left(\frac{8}{3} \hat{n}^{4}+\frac{14}{3} \hat{n}^{2} \Delta-2 \hat{n}^{2}-\frac{4}{3} \Delta^{2}-\Delta\right) n_{j}
$$


where the notations are the same as before, with $n_{j}$ and $\Delta_{j}$ being, respectively, the 'Liouville' charge and the total dimension of the insertion $\Psi_{j}\left(z_{j}\right)$, while $n_{i} \equiv \hat{n}$ and $\Delta_{i} \equiv \Delta$. Similarly to (4.6), the terms (4.21) have to vanish by virtue of a dressing prescription. The vanishing of $C_{i}$ gives

$$
\hat{n}^{2}=\frac{2 \delta}{3} \quad \text { or } \quad \delta=\frac{3}{8} .
$$

The first case reproduces the ' - ' case in (4.19) and (4.20). It will also agree with the dressing condition (4.12), i.e. $\Delta_{j}=-\frac{1}{2} Q n_{j} \equiv-\frac{1}{2} \sqrt{(1-d) / 3} n_{j}$ for $j=i$ once we choose

$$
\hat{n}=-\sqrt{\frac{1-d}{12}} .
$$

Then, the corresponding null vector takes the form

$$
\begin{aligned}
\left|\Upsilon_{22}\right\rangle= & \left(\widehat{L}_{-2} \widehat{L}_{-1}^{2}+2 \hat{n} I_{-1} \widehat{L}_{-2} \widehat{L}_{-1}+\frac{\hat{n}^{2}-\frac{1}{2}}{\hat{n}^{2}+1} \widehat{L}_{-3} \widehat{L}_{-1}-\frac{\hat{n}}{\hat{n}^{2}+1} I_{-1} \widehat{L}_{-1}^{3}\right. \\
& -\frac{\hat{n}^{4}+2 \hat{n}^{2}}{\hat{n}^{2}+1} \widehat{L}_{-2}^{2}+\frac{\frac{3}{2} \hat{n}^{2}}{\hat{n}^{2}+1} \widehat{L}_{-4}+\frac{\hat{n}^{3}+\frac{\hat{n}}{2}}{\hat{n}^{2}+1} I_{-3} \widehat{L}_{-1}-\frac{\frac{1}{4}}{\hat{n}^{2}+1} \widehat{L}_{-1}^{4} \\
& +\frac{\hat{n}}{\hat{n}^{2}+1} I_{-1}^{3} \widehat{L}_{-1}+\frac{\frac{1}{2}-\hat{n}^{2}}{\hat{n}^{2}+1} I_{-1}^{2} \widehat{L}_{-1}^{2}-\frac{\frac{3}{2} \hat{n}}{\hat{n}^{2}+1} I_{-2} \widehat{L}_{-1}^{2}-\frac{\hat{n}^{2}}{\hat{n}^{2}+1} I_{-1}^{2} \widehat{L}_{-2} \\
& \left.+\hat{n} I_{-2} \widehat{L}_{-2}+\frac{\hat{n}^{3}-\frac{1}{2} \hat{n}}{\hat{n}^{2}+1} I_{-1} \widehat{L}_{-3}-\frac{2 \hat{n}^{2}+\frac{1}{2}}{\hat{n}^{2}+1} I_{-2} I_{-1} \widehat{L}_{-1}\right)\left|\Psi_{22}\right\rangle,
\end{aligned}
$$

where $\widehat{L}_{m}$ is defined by the same formula (3.3) as before, but this time with

$$
Q=-2 \hat{n} \quad\left(l=2, l^{\prime}=2\right) .
$$

It is now straightforward to arrive at the decoupling equation of the type of (3.43) with 


$$
\begin{aligned}
& \widehat{\mathcal{O}}^{(22)}=-\frac{1}{4} \partial_{i}^{4}+\sum_{j \neq i} \frac{1}{z_{j}-z_{i}}\left\{n_{i} n_{j} \partial_{i}^{3}-\left(n_{i}^{2}+1\right) \partial_{j} \partial_{i}^{2}\right\}+\sum_{j \neq i} \frac{n_{i}^{3}+\frac{n_{i}}{2}}{\left(z_{j}-z_{i}\right)^{3}}\left(n_{i} \partial_{j}-n_{j} \partial_{i}\right) \\
+ & \sum_{j \neq i} \sum_{k \neq i} \frac{1}{\left(z_{j}-z_{i}\right)\left(z_{k}-z_{i}\right)}\left\{\left(\frac{1}{2}-n_{i}^{2}\right) n_{j} n_{k} \partial_{i}^{2}+2 n_{i} n_{j}\left(n_{i}^{2}+1\right) \partial_{i} \partial_{k}-\left(n_{i}^{2}+2\right) n_{i}^{2} \partial_{j} \partial_{k}\right\} \\
+\sum_{j \neq i} & \frac{1}{\left(z_{j}-z_{i}\right)^{2}}\left\{\left(\frac{1}{2}-n_{i}^{2}\right) \partial_{i} \partial_{j}+\frac{3}{2} n_{i} n_{j} \partial_{i}^{2}\right\}+\sum_{j \neq i k \neq i l \neq i} \sum_{i} \frac{n_{i} n_{k} n_{l}}{\left(z_{j}-z_{i}\right)\left(z_{k}-z_{i}\right)\left(z_{l}-z_{i}\right)}\left(n_{i} \partial_{j}-n_{j} \partial_{i}\right) \\
& +\sum_{j \neq i k \neq i} \sum_{i} \frac{1}{\left(z_{j}-z_{i}\right)\left(z_{k}-z_{i}\right)^{2}}\left\{\left(n_{i}^{2}+1\right) n_{k}\left(n_{i} \partial_{j}-n_{j} \partial_{i}\right)+\left(n_{i}^{2}-\frac{1}{2}\right) n_{j}\left(n_{i} \partial_{k}-n_{k} \partial_{i}\right)\right\} .
\end{aligned}
$$

We proceed with this operator in (5.49), where we establish its relation to the Virasoro constraints with the background charge given by eq. (4.26).

The other possibility in (4.19) is $\hat{n}^{2}=\frac{2 \delta}{3}+2=\frac{25-d}{12}$. Therefore, $\Delta=-\frac{d+11}{12}$, which can be reconciled with the dressing condition $\Delta=-\frac{1}{2} \sqrt{(1-d) / 3} \hat{n}$ only for the 'topological' 45alue $d=-2$ of the matter central charge [or, $\mathrm{c}=0$ according to (1.9)]. Then, $\delta=\frac{1-(-2)}{8}=\frac{3}{8}$, which is the other case encountered in (4.23). To summarize,

$$
\Delta=-\frac{3}{4}, \quad Q=1, \quad \hat{n}=\frac{3}{2}, \quad d=-2
$$

which thus applies to a particular type of matter, that consisting of spin-1 ghosts. Upon substituting (4.28) into the null vector (4.17), we find that the latter becomes identical to the '41' null vector (4.10) with $n_{i}$ fixed to its value (4.28), $n_{i}=\frac{3}{2}$. In fact, the question of when the ' 41 ' and ' 22 ' null vectors may coincide can be asked first for the 'bare' (purely minimal-model) null vectors. It is not difficult to see that this does indeed happen at the above value of the matter central charge $d=-2$ (hence the dimension $\left.\delta=\frac{3}{8}\right)$, but also at $d=28\left(\delta=-\frac{27}{8}\right)$. Then, the corresponding 'dressed' null vectors, (4.7) and (4.17), coincide as well, and we can ask next if the $I_{-1}^{4}$ term vanishes. When $d=-2$, the coefficient in front of the $I_{-1}^{4}$ term is proportional to

$$
\left(\hat{n}^{2}-\frac{9}{4}\right)\left(\hat{n}^{2}-\frac{1}{4}\right)
$$

while at $d=28$ this gets replaced by 


$$
\left(\hat{n}^{2}+\frac{9}{4}\right)\left(\hat{n}^{2}+\frac{1}{4}\right)
$$

Thus, the 'Liouville' charge becomes imaginary for $d=26-(-2)$. The imaginary unit can be absorbed into the 'liouville' current, thereby reversing its signature and giving it the rôle of a matter, while the matter itself, by the same procedure, becomes a 'Liouville' theory. Let us therefore concentrate on the case $(4.29), d=2$. Then the background charge $Q=\sqrt{\frac{1-d}{3}}=1$, and substituting this together with $\Delta=\delta-\frac{\hat{n}^{2}}{2}=\frac{3}{8}-\frac{\hat{n}^{2}}{2}$ into the dressing condition (4.12), we find

$$
\hat{n}=\left\{\begin{array}{l}
\frac{3}{2} \\
-\frac{1}{2}
\end{array}\right.
$$

which belongs to the roots of $(4.29)$. The upper value, which we have already met in $(4.28)$, satisfies also eq. (4.14), while the lower one agrees with both (4.15) and (4.26). We have thus seen how the 'exceptional' case (4.28), and the null vector considered at the end of Sect. 4.2.1, both fit into the pattern of 'intersections' between the ' 22 ' and ' 41 ' null vectors.

\section{$5 \quad$ From dressed null states to the Virasoro constraints}

We have considered in the previous sections the implications of the 'mirror' BRST invariance in the form of the 'Kontsevich-Miwa' dressed decoupling equations. In what follows we study its implications for correlators of ghost-independent chiral primary fields (hence, as before, of total ^-dimension zero). We can observe an interesting property by invoking the KontsevichMiwa transform, i.e. interpreting the $z_{j}$ and $n_{j}$ in (4.11), and (4.27) as the ingredients of the parametrization (1.2). Namely, we will show that the decoupling operators constructed according to the above recipe 'tend to' factorize through the Virasoro generators (1.1). The complete factorization occurs for the $(l, 1)$ decoupling equations, while for the $\left(l^{\prime}, l^{\prime \prime}\right)$ ones with either $l^{\prime}$ or $l^{\prime \prime} \neq 1$, there is in general an obstruction to the factorization. Even in the $(l, 1)$ case, the factorization property is by no means automatic, and does not apply for instance to the decoupling operator (4.4), without the relations (4.5) and (3.42).

More precisely, let us write the mapping (1.2) as $\mathcal{Z} \longrightarrow \mathcal{T}:\left(z_{j}\right) \mapsto\left(t_{r}=\frac{1}{r} \sum_{j \neq i} n_{j} z_{j}^{-r}\right)$. Then, at a point $\left(z_{j}\right)$ in the $\mathcal{Z}$ space, let $\partial / \partial z_{j}$ denote the directions along the fibre over 
$\left(t_{r}\right) \in \mathcal{T}$, so that the general infinitesimal displacements in $\mathcal{Z}$ can be written as $\partial_{j}=\frac{\partial}{\partial z_{j}}-$ $n_{j} \sum_{r \geq 1} z_{j}^{-r-1} \frac{\partial}{\partial t_{r}}$, where $\frac{\partial}{\partial z_{j}}$ does not affect the times. Then it turns out that, when acting on the function of only the times $t_{r}$, the $(l, 1)$ decoupling operators always factorize through the Virasoro generators (11.1) in which the background charge $2 \mathrm{~J}-1$ depends on the level $l$ chosen according to formula (1.5).

We are going to demonstrate this for the decoupling operators derived above.

\subsection{Level-2 decoupling equation as Virasoro constraints}

To transform the decoupling operator from the LHS of (3.29)

$$
\mathcal{T}=\frac{1}{2 n_{i}^{2}} \partial_{i}^{2}+\frac{1}{n_{i}} \sum_{j \neq i} \frac{1}{z_{j}-z_{i}}\left(n_{i} \partial_{j}-n_{j} \partial_{i}\right)
$$

into the $t$-variables, we can proceed in a naïve way by expressing the derivatives (when these act on functions depending only on the $t_{r}$ ) via

$$
\begin{aligned}
\frac{\partial}{\partial z_{i}}= & -n_{i} \sum_{r \geq 1} z_{i}^{-r-1} \frac{\partial}{\partial t_{r}}, \\
\frac{\partial^{2}}{\partial z_{i}^{2}}= & n_{i}^{2} \sum_{r, s \geq 1} z_{i}^{-r-s-2} \frac{\partial^{2}}{\partial t_{r} \partial t_{s}}+n_{i} \sum_{r \geq 1} z_{i}^{-r-2}(r+1) \frac{\partial}{\partial t_{r}}, \\
\frac{\partial^{3}}{\partial z_{i}^{3}}= & -n_{i}^{3} \sum_{q, r, s \geq 1} z_{i}^{-q-r-s-3} \frac{\partial^{3}}{\partial t_{q} \partial t_{r} \partial t_{s}}-3 n_{i}^{2} \sum_{r, s \geq 1}(r+1) z_{i}^{-r-s-3} \frac{\partial^{2}}{\partial t_{r} \partial t_{s}} \\
& -n_{i} \sum_{r \geq 1}(r+1)(r+2) z_{i}^{-r-3} \frac{\partial}{\partial t_{r}}
\end{aligned}
$$

(the third derivative is included for future use). Now, in the second term in $\mathcal{T}$, we substitute (5.2) and divide by $z_{j}-z_{i}$ to obtain

$$
\sum_{j \neq i} n_{j} \sum_{r \geq 1} \sum_{s=1}^{r+1} z_{j}^{-s} z_{i}^{-r+s-2} \frac{\partial}{\partial t_{r}} .
$$

Here, the sum over all $j$ gives $s t_{s}$ according to the Miwa transform (1.2); the missing term with $j=i$ should be added and subtracted. Thus 


$$
\sum_{j \neq i} \frac{1}{z_{j}-z_{i}}\left(n_{i} \partial_{j}-n_{j} \partial_{i}\right)=n_{i} \sum_{r \geq 1} \sum_{s=1}^{r+1} z_{i}^{s-r-2} s t_{s} \frac{\partial}{\partial t_{r}}-n_{i}^{2} \sum_{r \geq 1}(r+1) z_{i}^{-r-2} \frac{\partial}{\partial t_{r}}
$$

and therefore, finally,

$$
\mathcal{T}=\sum_{p \geq-1} z_{i}^{-p-2} \mathrm{~L}_{p}^{(2)}
$$

where the $\mathrm{L}_{n}^{(2)}$ are given by $(1.1)$ with

$$
2 \mathrm{~J}-1=\frac{1}{n_{i}}-2 n_{i} \quad(l=2) .
$$

It also follows that the Virasoro background charge $\mathrm{Q} \equiv 2 \mathrm{~J}-1$ equals $Q=\sqrt{(1-d) / 3}$, which is the matter background charge.

Therefore, the lowest-level decoupling equation is nothing but a linear combination of the $\{p \geq-1\}$-Virasoro generators. The inverse transformation, from the $\mathrm{L}_{p}$ into the decoupling equation, was carried out in ref. [21]. That is, starting with the 'energy-momentum tensor' $\sum_{p} z^{-p-2} \mathrm{~L}_{p}$ and evaluating at $z=z_{i}$ that part of it which is holomorphic at the infinity, we find that it equals the RHS of eq. (5.1). The existence of the inverse transform demonstrates that the (dressed!) correlation functions do indeed depend on the insertions point only through the time variables $t_{r}$.

\subsection{Virasoro constraints from level 3}

The decoupling equation, which is of the third order for level 3, can no longer just coincide with the Virasoro constraints, which are given by second-order differential operators. Yet the Virasoro constraints do emerge anyway. We will transform into the time variables the level-3 decoupling operator (3.44), $\widehat{\mathcal{O}} \equiv-n_{i} \mathcal{U}$,

$$
\begin{aligned}
\mathcal{U} \equiv & \\
-\frac{1}{n_{i}^{3}} \partial_{i}^{3} & +\left(1-\frac{1}{n_{i}^{2}}\right) \sum_{j \neq i} \frac{1}{\left(z_{j}-z_{i}\right)^{2}}\left(n_{i} \partial_{j}-n_{j} \partial_{i}\right)-\frac{1}{n_{i}^{2}} \sum_{j \neq i} \frac{1}{z_{j}-z_{i}}\left(2 n_{i} \partial_{j} \partial_{i}-3 n_{j} \partial_{i}^{2}\right) \\
& +\frac{2}{n_{i}} \sum_{j \neq i k \neq i} \sum_{k} \frac{n_{k}}{\left(z_{j}-z_{i}\right)\left(z_{k}-z_{i}\right)}\left(n_{i} \partial_{j}-n_{j} \partial_{i}\right),
\end{aligned}
$$


in two ways: the first one is a direct extension of the above level-2 approach, while the other allows further generalizations, which, in particular, will be used at level 4 .

\subsubsection{A naïve approach to level 3}

Proceeding as in 5.1, by directly dividing over $z_{j}-z_{i}$ as in (5.3), let us start with the second term on the RHS of (5.6). We find

$$
\begin{aligned}
& -\frac{1}{n_{i}^{2}} \sum_{j \neq i} \frac{1-n_{i}^{2}}{\left(z_{j}-z_{i}\right)^{2}}\left(n_{i} \partial_{j}-n_{j} \partial_{i}\right) \\
& =-\frac{1}{n_{i}} \sum_{j \neq i} \frac{1-n_{i}^{2}}{z_{j}-z_{i}} n_{j} \sum_{r \geq 1} \frac{z_{i}^{-r-1}-z_{j}^{-r-1}}{z_{j}-z_{i}} \frac{\partial}{\partial t_{r}} \\
& =\left(n_{i}-\frac{1}{n_{i}}\right)\left\{\sum_{j \neq i} \frac{n_{j}}{z_{j}-z_{i}} \sum_{r \geq 1} \sum_{s=1}^{r+1}\left(z_{j}^{-s}-z_{i}^{-s}\right) z_{i}^{-r+s-2} \frac{\partial}{\partial t_{r}}+\sum_{j \neq i} \frac{n_{j}}{z_{j}-z_{i}} \sum_{r \geq 1} z_{i}^{-r-2}(r+1) \frac{\partial}{\partial t_{r}}\right\} .
\end{aligned}
$$

The first term on the RHS of the last equation, in its own turn, is equal to

$$
-\left(n_{i}-\frac{1}{n_{i}}\right) \sum_{r \geq 1} \sum_{q=1}^{r+1}(r+2-q) z_{i}^{q-r-3} q t_{q} \frac{\partial}{\partial t_{r}}-\left(1-n_{i}^{2}\right) \sum_{r \geq 1} \sum_{q=1}^{r+1}(r+2-q) z_{i}^{-r-3} \frac{\partial}{\partial t_{r}}
$$

and therefore eq. (5.7) becomes

$$
\begin{aligned}
& \left(\frac{1}{n_{i}}-n_{i}\right) \sum_{p \geq-1} \sum_{\substack{q \geq 1 \\
q+p \geq 1}}(p+2) z_{i}^{-p-3} q t_{q} \frac{\partial}{\partial t_{p+q}} \\
& +\left(n_{i}^{2}-1\right) \sum_{p \geq 1} \frac{1}{2}(p+1)(p+2) z_{i}^{-p-3} \frac{\partial}{\partial t_{p}}+\left(n_{i}-\frac{1}{n_{i}}\right) \sum_{j \neq i} \frac{n_{j}}{z_{j}-z_{i}} \sum_{p \geq 1} z_{i}^{-p-2}(p+1) \frac{\partial}{\partial t_{p}} .
\end{aligned}
$$

Similarly, in the last term in (5.6) we can also divide by $z_{j}-z_{i}$ in the following way:

$$
\begin{aligned}
& \frac{2}{n_{i}} \sum_{k \neq i} \frac{n_{k}}{z_{k}-z_{i}} \sum_{j \neq i} n_{i} n_{j} \sum_{r \geq 1} \frac{z_{i}^{-r-1}-z_{j}^{-r-1}}{z_{j}-z_{i}} \frac{\partial}{\partial t_{r}} \\
& =2 \sum_{j \neq i} \frac{n_{j}}{z_{j}-z_{i}} \sum_{\substack { p \geq-1 \\
\begin{subarray}{c}{1 \\
p>s \geq 1{ p \geq - 1 \\
\begin{subarray} { c } { 1 \\
p > s \geq 1 } }\end{subarray}} s t_{s} z_{i}^{-p-2} \frac{\partial}{\partial t_{p+s}}-2 n_{i} \sum_{j \neq i} \frac{n_{j}}{z_{j}-z_{i}} \sum_{p \geq 1}(p+1) z_{i}^{-p-2} \frac{\partial}{\partial t_{p}} .
\end{aligned}
$$


Further, the term with second derivatives in (5.6) equals

$$
\begin{aligned}
& 2 \sum_{p \geq 0} z_{i}^{-p-3} \sum_{\substack{s=-1 \\
q \geq 1}}^{p-1} q t_{q} \frac{\partial^{2}}{\partial t_{p-s} \partial t_{q+s}}-2 n_{i} \sum_{p \geq 2} z_{i}^{-p-3} \sum_{s=1}^{p-1}(s+1) \frac{\partial^{2}}{\partial t_{p-s} \partial t_{s}} \\
& -\frac{1}{n_{i}} \sum_{j \neq i} \frac{1}{z_{j}-z_{i}}\left(-n_{i} n_{j} \sum_{r \geq 1 s \geq 1} z_{i}^{-r-s-2} \frac{\partial^{2}}{\partial t_{r} \partial t_{s}}-3 n_{j} \sum_{r \geq 1}(r+1) z_{i}^{-r-2} \frac{\partial}{\partial t_{r}}\right) .
\end{aligned}
$$

Substituting also the expression (5.2) for the third derivative present in the decoupling operator (5.6) and collecting everything together we find

$$
\begin{aligned}
\mathcal{U} & =\sum_{q, r, s \geq 1} z_{i}^{-q-r-s-3} \frac{\partial^{3}}{\partial t_{q} \partial t_{r} \partial t_{s}}+\left(\frac{3}{n_{i}}-2 n_{i}\right) \sum_{r, s \geq 1} z_{i}^{-r-s-3}(r+1) \frac{\partial^{2}}{\partial t_{r} \partial t_{s}} \\
+ & \left(\frac{1}{n_{i}^{2}}+\frac{n_{i}^{2}}{2}-\frac{1}{2}\right) \sum_{r \geq 1}(r+1)(r+2) z_{i}^{-r-3} \frac{\partial}{\partial t_{r}}+\frac{1-n_{i}^{2}}{n_{i}} \sum_{\substack{p \geq-1 \\
q \geq 1-p}} z_{q}^{-p-3}(p+2) q t_{q} \frac{\partial}{\partial t_{p+q}} \\
+ & 2 \sum_{p \geq 0} z_{i}^{-p-3} \sum_{s=-1}^{p-1} \sum_{\substack{q \geq 1 \\
q \geq 1-s}} q t_{q} \frac{\partial^{2}}{\partial t_{p-s} \partial t_{q+s}}+2 \sum_{j \neq i} \frac{n_{j}}{z_{j}-z_{i}} \sum_{p \geq-1}\left(z_{i}^{-p-2}-z_{j}^{-p-2}\right) \mathrm{L}_{p}^{(3)} \\
& +2 \sum_{j \neq i} \frac{n_{j}}{z_{j}-z_{i}} \sum_{p \geq-1} z_{j}^{-p-2} \mathrm{~L}_{p}^{(3)},
\end{aligned}
$$

where

$$
\begin{aligned}
\mathrm{L}_{p \geq 1}^{(3)} & =\frac{1}{2} \sum_{s=1}^{p-1} \frac{\partial^{2}}{\partial t_{p-s} \partial t_{s}}+\sum_{s \geq 1} s t_{s} \frac{\partial}{\partial t_{p+s}}+\frac{2-n_{i}^{2}}{2 n_{i}}(p+1) \frac{\partial}{\partial t_{p}} \\
\mathrm{~L}_{0}^{(3)} & =\sum_{s \geq 1} s t_{s} \frac{\partial}{\partial t_{s}} \\
\mathrm{~L}_{-1}^{(3)} & =\sum_{s \geq 1}(s+1) t_{s+1} \frac{\partial}{\partial t_{s}}
\end{aligned}
$$

are Virasoro generators, which differ, however, from the ones we have encountered in (5.4), (5.5). That is, the 'improvement' term is such that the spin $\mathrm{J}$ is related to $n_{i}$ via

$$
\frac{2}{n_{i}}-n_{i}=2 \mathrm{~J}-1 \quad(l=3) .
$$


Note that the last term has been added to and subtracted from the RHS of (5.12). With the combination $\left(z_{i}^{-p-2}-z_{j}^{-p-2}\right) /\left(z_{j}-z_{i}\right)$ we proceed as above, and in this way get new terms

$$
2 \sum_{p \geq-1} \sum_{s=1}^{p+2} z_{i}^{-p+s-3} s t_{s} \mathrm{~L}_{p}^{(3)}-2 n_{i} \sum_{p \geq-1} z_{i}^{-p-3}(p+2) \mathrm{L}_{p}^{(3)}
$$

Therefore,

$$
\mathcal{U}=\sum_{p \geq-2} z_{i}^{-p-3} \mathrm{U}_{p}+2 \sum_{j \neq i} \frac{n_{j}}{z_{j}-z_{i}} \sum_{p \geq-1} z_{j}^{-p-2} \mathrm{~L}_{p}^{(3)}
$$

where

$$
\begin{aligned}
\mathrm{U}_{-2}= & 2 \sum_{r \geq 1} r t_{r} \mathrm{~L}_{r-2}^{(3)} \\
\mathrm{U}_{-1}= & 2 \sum_{r \geq 1} r t_{r} \mathrm{~L}_{r-1}^{(3)}-2 n_{i} \mathrm{~L}_{-1}^{(3)}+\frac{1-n_{i}^{2}}{n_{i}} \sum_{r \geq 2} r t_{r} \frac{\partial}{\partial t_{r-1}} \\
\mathrm{U}_{0}= & 2 \sum_{r \geq 1} r t_{r} \mathrm{~L}_{r}^{(3)}-4 n_{i} \mathrm{~L}_{0}^{(3)}+2 \frac{1-n_{i}^{2}}{n_{i}} \sum_{r \geq 1} r t_{r} \frac{\partial}{\partial t_{r}}+2 \sum_{r \geq 2} r t_{r} \frac{\partial^{2}}{\partial t_{1} \partial t_{r-1}} \\
\mathrm{U}_{p \geq 1}= & 2 \sum_{r \geq 1} r t_{r} \mathrm{~L}_{r+p}^{(3)}-2(p+2) n_{i} \mathrm{~L}_{p}^{(3)}+(p+2) \frac{1-n_{i}^{2}}{n_{i}} \sum_{r \geq 1} r t_{r} \frac{\partial}{\partial t_{r+p}}+2 \sum_{s=-1}^{p-1} \sum_{\substack{r \geq 1 \\
r \geq 1}} r t_{r} \frac{\partial^{2}}{\partial t_{p-s} \partial t_{r+s}} \\
& +(p+1)(p+2)\left(\frac{1}{n_{i}^{2}}+\frac{n_{i}^{2}}{2}-\frac{1}{2}\right) \frac{\partial}{\partial t_{p}}+\left(\frac{3}{n_{i}}-2 n_{i}\right) \sum_{r=1}^{p-1}(r+1) \frac{\partial^{2}}{\partial t_{r} \partial t_{p-r}}+\sum_{\substack{q, r, s \geq 1 \\
q+r+s=p}} \frac{\partial^{3}}{\partial t_{t} \partial t_{r} \partial t_{s}}
\end{aligned}
$$

We can further rewrite the generic $U_{p}$ as

$$
\mathrm{U}_{p}=2 \sum_{q=-1}^{p-1} \frac{\partial}{\partial t_{p-q}} \circ \mathrm{L}_{q}^{(3)}+2 \sum_{r \geq 1} r t_{r} \mathrm{~L}_{r+p}^{(3)}+(p+2)\left(\frac{1}{n_{i}}-3 n_{i}\right) \mathrm{L}_{p}^{(3)}, \quad p \geq-2
$$

which shows that the $\mathrm{U}_{n}$ factorize through the Virasoro generators, and thus the $\mathrm{U}_{n}$ will annihilate the tau function once the $\mathrm{L}_{p}^{(3)}$ do:

$$
\mathrm{L}_{p}^{(3)} \tau=0, \quad p \geq-1
$$


Thus the Virasoro generators with the background charge tuned according to (5.14) are hidden in the dressed decoupling equation (or, in the BRST-invariance condition in our realization of the topological algebra). It follows from (5.14) and (3.39) that (as was the case at level 2) the minimal matter background charge coincides with the one involved in the Virasoro generators, $\sqrt{(1-d) / 3} \equiv Q=2 \mathrm{~J}-1$.

The structure of the operators $\mathrm{U}_{p}$ can be described in slightly more 'invariant' terms, as follows. Introduce a current $\mathbf{I}(z)=\sum_{m \in \mathbf{Z}} z^{-m-1} \mathbf{I}_{m}$, where田

$$
\begin{gathered}
\mathrm{I}_{m>0}=\frac{\partial}{\partial t_{m}}, \\
\mathrm{I}_{m<0}=-m t_{-m},
\end{gathered} \Longrightarrow\left\{\begin{array}{l}
{\left[\mathrm{I}_{m}, \mathrm{I}_{n}\right]=m \delta_{m+n, 0}} \\
{\left[\mathrm{~L}_{m}^{(3)}, \mathrm{I}_{n}\right]=-n \mathrm{I}_{m+n} .}
\end{array}\right.
$$

Then,

$$
\mathrm{U}_{p}=2 \sum_{n \leq p+1} \mathrm{I}_{n} \mathrm{~L}_{p-n}^{(3)}+(p+2)\left(\frac{1}{n_{i}}-3 n_{i}\right) \mathrm{L}_{p}^{(3)}
$$

Obviously, any commutator of the U's will always factorize in a similar way, with the L's on the right.

As the constraints reduce to the Virasoro ones, their consistency with the KP flows is achieved trivially by virtue of the results of [47]. Therefore, the initial decoupling equation also must be consistent with the Miwa-transformed version of the KP evolutions.

The generators (5.17) were first derived from the dressed decoupling equation in [46] although they were misinterpreted there. As we see, the actual mechanism is that the thirdorder differential operator factorizes through the Virasoro generators. This property persists at higher levels, but already at level 4 the factorization requires a more 'invariant' derivation, which will be first demonstrated below on the already familiar level-3 example.

\subsubsection{Level-3 factorization once more}

The above derivation of eq. (5.16), first carried out in ref. [46], might be fraught with an ambiguity, as the times $t_{r}$ have been viewed in the formulae such as (5.17) as being independent

\footnotetext{
${ }^{15} \mathbf{I}_{0}$, which is a c-number, is the 'zeroth time' of the KP hierarchy that distinguishes between its different Schlesinger-transformed copies [48 (or essentially the parameter $N$ from ref. [47]). However, its value is irrelevant for the following and can be assumed to be equal to zero.
} 
of the $z_{j}$; however, it was not clear from the above whether indeed all the $z$ 's should be placed to the left of the $\partial / \partial t_{r}$, as was the case in (5.16). At higher levels, a similar ambiguitiy becomes more severe, so we now present an alternative derivation of (5.16), which avoids the ordering problem (while leading to the same result).

Our starting point will be the previously established eq. (5.4), which we rewrite in the form

$$
\begin{aligned}
\partial_{i}^{2}= & -2 n_{i} \sum_{j \neq i} \frac{1}{z_{j}-z_{i}}\left(n_{i} \partial_{j}-n_{j} \partial_{i}\right) \\
& +2 n_{i}^{2} \sum_{p \geq-1} z_{i}^{-p-2} \mathrm{~L}_{p}^{(3)}-\left(n_{i}+n_{i}^{3}\right) \sum_{r \geq 1} z_{i}^{-r-2}(r+1) \frac{\partial}{\partial t_{r}}
\end{aligned}
$$

where we have anticipated the result by identifying on the RHS the level-3 Virasoro generators (5.13).

Now, turning to (5.6) we substitute (5.22) into the term $-\frac{1}{n_{i}^{3}} \partial_{i}^{3}=-\frac{1}{n_{i}^{3}} \partial_{i} \circ \partial_{i}^{2}$ and then again into the resulting expression $\sum_{j \neq i} \frac{n_{j}}{z_{j}-z_{i}} \partial_{i}^{2}$. We get in this way

$$
\begin{aligned}
\mathcal{U}= & \partial_{i} \circ\left\{-\frac{2}{n_{i}} \sum_{p \geq-1} z_{i}^{-p-2} \mathrm{~L}_{p}^{(3)}+\left(\frac{1}{n_{i}^{2}}+1\right) \sum_{r \geq 1} z_{i}^{-r-2}(r+1) \frac{\partial}{\partial t_{r}}\right\} \\
& +2 \sum_{j \neq i} \frac{n_{j}}{z_{j}-z_{i}} \sum_{p \geq-1} z_{i}^{-p-2} \mathrm{~L}_{p}^{(3)}+\frac{1}{2}\left(n_{i}^{2}+1\right) \sum_{r \geq 1}(r+1)(r+2) z_{i}^{-r-3} \frac{\partial}{\partial t_{r}} \\
& -\left(n_{i}+\frac{1}{n_{i}}\right) \sum_{r \geq 1} \sum_{s=1}^{r+1}(r+2-s) s t_{s} z_{i}^{s-r-3} \frac{\partial}{\partial t_{r}} .
\end{aligned}
$$

Here, $\partial_{i}$ is a differential operator, composed with the operator inside the braces, which can be represented as $\partial_{i}=\frac{\partial}{\partial z_{i}}-n_{i} \sum_{r \geq 1} z_{i}^{-r-1} \frac{\partial}{\partial t_{r}}$, where $\frac{\partial}{\partial z_{i}}$ does not affect the $t_{r}$ and acts only on the explicit occurrences of $z_{i}$. The operator $\mathcal{U}$ is understood to act on functions which depend on only the times $t_{r}$. Thus,

$$
\begin{aligned}
\partial_{i} \circ\{\ldots\}= & 2 \sum_{r \geq 1} \sum_{p \geq-1} z_{i}^{-r-p-3} \frac{\partial}{\partial t_{r}} \circ \mathbf{L}_{p}^{(3)}+\left(\frac{1}{2 n_{i}}-\frac{n_{i}}{2}\right) \sum_{r, s \geq 1} z_{i}^{-r-s-3}(r+s+2) \frac{\partial^{2}}{\partial t_{r} \partial t_{s}} \\
& +\frac{2}{n_{i}} \sum_{r \geq 1} \sum_{s=1}^{r+1}(r+2-s) s t_{s} z_{i}^{s-r-3} \frac{\partial}{\partial t_{r}}+\left(\frac{1}{n_{i}^{2}}-2\right) \sum_{r \geq 1}(r+1)(r+2) z_{i}^{-r-3} \frac{\partial}{\partial t_{r}} .
\end{aligned}
$$


Inserting (5.24) into the above expression for $\mathcal{U}$, we find

$$
\begin{aligned}
\mathcal{U}= & 2 \sum_{r \geq 1} \sum_{p \geq-1} z_{i}^{-r-p-3} \frac{\partial}{\partial t_{r}} \circ \mathrm{L}_{p}^{(3)}+2 \sum_{j \neq i} \frac{n_{j}}{z_{j}-z_{i}} \sum_{p \geq-1} z_{i}^{-p-2} \mathrm{~L}_{p}^{(3)} \\
& +\left(\frac{1}{n_{i}}-n_{i}\right) \sum_{p \geq-1}(p+2) z_{i}^{-p-3} \mathrm{~L}_{p}^{(3)}
\end{aligned}
$$

which is the same as (5.16), (5.18). Now we will see how the same approach works at level 4 .

\subsection{Factorization at level 4}

Recall that we had in Sect. 4 more than one relevant null vectors at level 4 . We will use the method of section 5.2 .2 to try to obtain factorization of the decoupling operators, after the Kontsevich-Miwa transform, into an expression of the form

$$
\widehat{\mathcal{O}}=\mathcal{A} \circ \sum_{p \geq-1} z_{i}^{-p-2} \mathrm{~L}_{p}^{(*)}
$$

where $\mathrm{L}^{(*)}$ are Virasoro generators with the expected background charges: either (4.14) for the ' 41 ' case, or, for the decoupling operator (4.27), the background charge $\mathrm{Q}=-2 n_{i}$, as suggested by (4.26). The 'regular' case, which reproduces the features we have observed for the '21' and '31' operators, is provided by the '41' null vector (4.10).

\subsubsection{Virasoro constraints from the ' 41 ' decoupling operator}

The factorization of the decoupling operator (4.11) will come about as a result of an interplay of the various coefficients, which appears rather miraculous in the present straightforward approach, and takes some work to be established. Our final result for the Kontsevich-Miwatransformed decoupling operator (4.11) is given in eq. (5.46) below. Although a result as simple must have a simpler derivation, all that we can suggest at the moment is the following collection of technicalities.

To simplify things, we will utilize in the derivation both the level-2 and level-3 factorization identities found previously. It is extremely useful to rewrite the level-3 one, eq. (5.18), as 


$$
\begin{aligned}
\partial_{i}^{3}= & 2 n_{i}^{2} \nabla_{i} \circ \mathrm{V}^{(3)}\left(z_{i}\right)-\left(n_{i}^{4}+n_{i}^{2}\right) \mathrm{V}^{(3)^{\prime}}\left(z_{i}\right)+\left(n_{i}^{3}-n_{i}\right) \sum_{j \neq i} \frac{1}{\left(z_{j}-z_{i}\right)^{2}}\left(n_{i} \partial_{j}-n_{j} \partial_{i}\right) \\
& -n_{i} \sum_{j \neq i} \frac{1}{z_{j}-z_{i}}\left(2 n_{i} \partial_{i} \partial_{j}-3 n_{j} \partial_{i}^{2}\right)+2 n_{i}^{2} \sum_{j \neq i k \neq i} \frac{n_{k}}{\left(z_{j}-z_{i}\right)\left(z_{k}-z_{i}\right)}\left(n_{i} \partial_{j}-n_{j} \partial_{i}\right),
\end{aligned}
$$

where we have introduced the compact notations

$$
\mathrm{V}^{(3)}\left(z_{i}\right)=\sum_{p \geq-1} z_{i}^{-p-2} \mathrm{~L}_{p}^{(3)}
$$

and

$$
\mathrm{V}^{(3)^{\prime}}\left(z_{i}\right)=\sum_{p \geq-1} z_{i}^{-p-3}(-p-2) \mathbf{L}_{p}^{(3)}
$$

for linear combinations of the level-3 Virasoro generators (5.13), while

$$
\nabla_{i}=\partial_{i}-n_{i} \sum_{j \neq i} \frac{n_{j}}{z_{j}-z_{i}}
$$

is another very useful combination.

Let us call inv the space of functions which depend on the $z_{j}$ only through the times $t_{r}$. It is understood that all the operators are considered on this subspace; thus, we are in fact evaluating $\left.\widehat{\mathcal{O}}^{(4)}\right|_{\text {inv }}$, which, however, will not be indicated explicitly.

We substitute (5.27) into (4.11), expressing $\partial_{i}^{4}$ as $\partial_{i} \circ \partial_{i}^{3}$. Then, we get other $\partial_{i}^{3}$-terms sitting over a pole in $z_{j}-z_{i}$. Substituting (5.27) again, we then collect together all the $\partial_{i}^{2}$-terms and use for these the previous identity (5.4), rewritten in the current notations as

$$
\partial_{i}^{2}=-2 n_{i} \sum_{j \neq i} \frac{1}{z_{j}-z_{i}}\left(n_{i} \partial_{j}-n_{j} \partial_{i}\right)+2 n_{i}^{2} \mathrm{\vee}^{(2)}\left(z_{i}\right)
$$

where $\mathrm{V}^{(2)}\left(z_{i}\right) \equiv \mathcal{T}$ is the same as (5.28) for the level-2 Virasoro generators [i.e. those with the background charge (5.5)]. By these manipulations, we bring the decoupling operator (4.11) to the form 


$$
\begin{aligned}
\widehat{\mathcal{O}}^{(4)}= & \left(-\frac{8 n_{i}^{3}}{45}-\frac{11 n_{i}}{30}+\frac{3}{10 n_{i}}\right) \sum_{j \neq i} \frac{1}{\left(z_{j}-z_{i}\right)^{3}}\left(n_{i} \partial_{j}-n_{j} \partial_{i}\right) \\
& +\left(\frac{n_{i}^{2}}{12}+\frac{7}{20}\right)\left\{\sum_{j \neq i} \frac{1}{\left(z_{j}-z_{i}\right)^{2}} \partial_{i} \partial_{j}\right. \\
& -\sum_{j \neq i k \neq i} \sum_{k} \frac{n_{k}}{\left(z_{j}-z_{i}\right)^{2}\left(z_{k}-z_{i}\right)}\left(n_{i} \partial_{j}-n_{j} \partial_{i}\right) \\
& +\left(-\frac{13 n_{i}^{3}}{30}-\frac{6 n_{i}}{5}\right) \sum_{j \neq i} \frac{n_{k}}{\left(z_{j}-z_{i}\right)^{2}} \mathrm{~V}^{(2)}\left(z_{i}\right) \\
& -\frac{n_{i}^{2}}{5} \sum_{j \neq i} \frac{1}{z_{j}-z_{i}} \partial_{j} \circ \mathrm{V}^{(2)}\left(z_{i}\right)+\frac{n_{i}^{2}}{10} \sum_{j \neq i k \neq i} \sum_{i} \frac{n_{j} n_{k}}{\left(z_{j}-z_{i}\right)\left(z_{k}-z_{i}\right)} \mathrm{V}^{(2)}\left(z_{i}\right) \\
& \left.\left.-\frac{9}{10} \nabla_{i} \circ \nabla_{i} \circ \partial_{i}\right)\right\}
\end{aligned}
$$

Now, in the terms that do not contain an explicit $\mathrm{V}\left(z_{i}\right)$ we use an obvious identity

$$
\begin{gathered}
n_{i}\left(\sum_{j \neq i} \frac{1}{\left(z_{j}-z_{i}\right)^{2}} \partial_{i} \partial_{j}-\sum_{j \neq i k \neq i} \frac{n_{k}}{\left(z_{j}-z_{i}\right)^{2}\left(z_{k}-z_{i}\right)}\left(n_{i} \partial_{j}-n_{j} \partial_{i}\right)\right. \\
\left.+2 \sum_{j \neq i k \neq i} \frac{n_{k}}{\left(z_{j}-z_{i}\right)\left(z_{k}-z_{i}\right)^{2}}\left(n_{i} \partial_{j}-n_{j} \partial_{i}\right)\right) \\
=\nabla_{i} \circ \sum_{j \neq i} \frac{1}{\left(z_{j}-z_{i}\right)^{2}}\left(n_{i} \partial_{j}-n_{j} \partial_{i}\right)+2 \sum_{j \neq i} \frac{n_{j}}{\left(z_{j}-z_{i}\right)^{2}} \mathrm{~V}^{(2)}\left(z_{i}\right)-2 \sum_{j \neq i} \frac{1}{\left(z_{j}-z_{i}\right)^{3}}\left(n_{i} \partial_{j}-n_{j} \partial_{i}\right) .
\end{gathered}
$$

Another trivial but useful rearrangement reads

$$
n_{i}^{2} \sum_{j \neq i k \neq i} \frac{n_{j} n_{k}}{\left(z_{j}-z_{i}\right)\left(z_{k}-z_{i}\right)}=\nabla_{i}^{2}-\partial_{i}^{2}+2 n_{i} \sum_{j \neq i} \frac{n_{j}}{z_{j}-z_{i}} \partial_{i}+n_{i} \sum_{j \neq i} \frac{n_{j}}{\left(z_{j}-z_{i}\right)^{2}} .
$$

Using these allows us to arrive at 


$$
\begin{aligned}
\widehat{\mathcal{O}}^{(4)}= & \left(\frac{4 n_{i}^{2}}{15}+\frac{2}{5}\right)\left(-\frac{1}{n_{i}}-\frac{2 n_{i}}{3}\right) \sum_{j \neq i} \frac{1}{\left(z_{j}-z_{i}\right)^{3}}\left(n_{i} \partial_{j}-n_{j} \partial_{i}\right) \\
& +\left(\frac{n_{i}}{12}+\frac{7}{20 n_{i}}\right) \nabla_{i} \circ \sum_{j \neq i} \frac{1}{\left(z_{j}-z_{i}\right)^{2}}\left(n_{i} \partial_{j}-n_{j} \partial_{i}\right) \\
& +\left(-\frac{4 n_{i}^{3}}{15}-\frac{2 n_{i}}{5}\right) \sum_{j \neq i} \frac{n_{j}}{\left(z_{j}-z_{i}\right)^{2}} \mathrm{~V}^{(2)}\left(z_{i}\right)-\frac{n_{i}}{5} \sum_{j \neq i} \frac{1}{z_{j}-z_{i}}\left(n_{i} \partial_{j}-n_{j} \partial_{i}\right) \circ \mathrm{V}^{(2)}\left(z_{i}\right) \\
& +\frac{9}{20}\left(n_{i}^{2}+1\right) \nabla_{i} \circ \mathrm{V}^{(3)^{\prime}}\left(z_{i}\right)-\frac{1}{10} \partial_{i}^{2} \circ \mathrm{V}^{(2)}\left(z_{i}\right) \\
& +\left(\frac{n_{i}}{12}+\frac{7}{20 n_{i}}\right) \nabla_{i}^{2} \circ \sum_{r \geq 1} z_{i}^{-r-2}(r+1) \frac{\partial}{\partial t_{r}}-\frac{4}{5} \nabla_{i}^{2} \circ \mathrm{V}^{(4)}\left(z_{i}\right) .
\end{aligned}
$$

The last two terms here are just a rewriting of

$$
\frac{1}{10} \nabla_{i}^{2} \circ \mathrm{V}^{(2)}\left(z_{i}\right)-\frac{9}{10} \nabla_{i}^{2} \circ \mathrm{V}^{(3)}\left(z_{i}\right)
$$

and we have introduced the 'level-4' Virasoro generators as suggested by formula (4.14),

$$
\mathrm{L}_{r \geq 1}^{(4)}=\frac{1}{2} \sum_{s=1}^{r-1} \frac{\partial^{2}}{\partial t_{s} \partial t_{r-s}}+\sum_{s \geq 1} s t_{s} \frac{\partial}{\partial t_{s+r}}+\left(\frac{3}{2 n_{i}}-\frac{n_{i}}{3}\right)(r+1) \frac{\partial}{\partial t_{r}}
$$

We thus get in (5.35) two terms with the same coefficient $\left(\frac{n_{i}}{12}+\frac{7}{20 n_{i}}\right)$, and to add these together we use the identity

$$
\begin{aligned}
& \sum_{j \neq i} \frac{1}{\left(z_{j}-z_{i}\right)^{2}}\left(n_{i} \partial_{j}-n_{j} \partial_{i}\right)+\nabla_{i} \circ \sum_{r \geq 1} z_{i}^{-r-2}(r+1) \frac{\partial}{\partial t_{r}} \\
& =n_{i} \mathrm{~V}^{(3)^{\prime}}\left(z_{i}\right) \\
& =n_{i} \mathrm{~V}^{(4)^{\prime}}\left(z_{i}\right)+\left(\frac{n_{i}^{2}}{6}+\frac{1}{2}\right) \sum_{r \geq 1}(r+1)(r+2) z_{i}^{-r-3} \frac{\partial}{\partial t_{r}}
\end{aligned}
$$

Similarly, in the first term in (5.35) we use the identity 


$$
\begin{aligned}
\sum_{j \neq i} \frac{1}{\left(z_{j}-z_{i}\right)^{3}}\left(n_{i} \partial_{j}-n_{j} \partial_{i}\right)= & n_{i} \sum_{j \neq i} \frac{n_{j}}{\left(z_{j}-z_{i}\right)^{2}} \sum_{r \geq 1}(r+1) z_{i}^{-r-2} \frac{\partial}{\partial t_{r}}+\frac{n_{i}}{2} \mathrm{~V}^{(4)^{\prime \prime}}\left(z_{i}\right) \\
& +\frac{1}{2} \nabla_{i} \circ \sum_{r \geq 1}(r+1)(r+2) z_{i}^{-r-3} \frac{\partial}{\partial t_{r}} \\
& -\frac{n_{i}}{2} \sum_{r, s \geq 1}(r+1)(s+1) z_{i}^{-r-s-4} \frac{\partial^{2}}{\partial t_{r} \partial t_{s}} \\
& -\frac{1}{4} \sum_{r \geq 1}(r+1)(r+2)(r+3) z_{i}^{-r-4} \frac{\partial}{\partial t_{r}},
\end{aligned}
$$

where

$$
\mathrm{V}^{(4)^{\prime \prime}}\left(z_{i}\right)=\sum_{p \geq-1} z_{i}^{-p-4}(p+2)(p+3) \mathbf{L}_{p}^{(4)}
$$

As a result, the operator $\widehat{\mathcal{O}}^{(4)}$ becomes

$$
\begin{aligned}
\widehat{\mathcal{O}}^{(4)}= & \left(\frac{n_{i}^{2}}{12}+\frac{7}{20}\right) \nabla_{i} \circ \mathrm{V}^{(4)^{\prime}}\left(z_{i}\right)+\left(-\frac{4 n_{i}^{3}}{15}-\frac{2 n_{i}}{5}\right) \sum_{j \neq i} \frac{n_{j}}{\left(z_{j}-z_{i}\right)^{2}} \mathrm{~V}^{(4)}\left(z_{i}\right) \\
& +\frac{9}{20}\left(n_{i}^{2}+1\right) \nabla_{i} \circ \mathrm{V}^{(3)^{\prime}}\left(z_{i}\right)-\frac{4}{5} \nabla_{i}^{2} \circ \mathrm{V}^{(4)}\left(z_{i}\right) \\
& -\frac{n_{i}}{5} \sum_{j \neq i} \frac{1}{z_{j}-z_{i}}\left(n_{i} \partial_{j}-n_{j} \partial_{i}\right) \circ \mathrm{V}^{(2)}\left(z_{i}\right)-\frac{1}{10} \partial_{i}^{2} \circ \mathrm{V}^{(2)}\left(z_{i}\right) \\
& +\frac{n_{i}}{5}\left(\frac{1}{n_{i}}+\frac{2 n_{i}}{3}\right)^{2}\left\{-n_{i} \mathrm{~V}^{(4)^{\prime \prime}}\left(z_{i}\right)+n_{i} \sum_{r, s \geq 1}(r+1)(s+1) z_{i}^{-r-s-4} \frac{\partial^{2}}{\partial t_{r} \partial t_{s}}\right. \\
& +\left(-\frac{1}{2} \sum_{r \geq 1}(r+1)(r+2)(r+3) z_{i}^{-r-4} \frac{\partial}{\partial n_{r}}\right\} \\
& \left.-\frac{n_{i}}{6}-\frac{1}{40 n_{i}}\right) \nabla_{i} \circ \sum_{r \geq 1}(r+1)(r+2) z_{i}^{-r-3} \frac{\partial}{\partial t_{r}} .
\end{aligned}
$$

Now, in the term $\frac{9}{20}\left(n_{i}^{2}+1\right) \nabla_{i} \circ \mathrm{V}^{(3)^{\prime}}\left(z_{i}\right)$ we replace $\mathrm{L}_{p}^{(3)}$ with $\mathrm{L}_{p}^{(4)}$; the difference then adds up with the last term in (5.41) to produce the contribution

$$
\left(\frac{2 n_{i}}{15}+\frac{1}{5 n_{i}}\right) \nabla_{i} \circ \sum_{r \geq 1}(r+1)(r+2) z_{i}^{-r-3} \frac{\partial}{\partial t_{r}} .
$$


On the other hand, we have in (5.41)

$$
\begin{aligned}
-\frac{n_{i}}{5} \sum_{j \neq i} \frac{1}{z_{j}-z_{i}}\left(n_{i} \partial_{j}-n_{j} \partial_{i}\right) \circ \mathrm{V}^{(2)}\left(z_{i}\right)= & -\frac{n_{i}}{5} \sum_{p \geq-1} z_{i}^{-p-2} \sum_{j \neq i} \frac{1}{z_{j}-z_{i}}\left(n_{i} \partial_{j}-n_{j} \partial_{i}\right) \circ \mathrm{L}_{p}^{(2)} \\
& +\frac{n_{i}}{5} \sum_{j \neq i} \frac{n_{j}}{z_{j}-z_{i}} \mathrm{~V}^{(2)^{\prime}}\left(z_{i}\right) .
\end{aligned}
$$

The last piece here combines with that part of (5.42) that sits over the pole coming from the definition of $\nabla_{i}$, to produce precisely a $\bigvee^{(4)^{\prime}}\left(z_{i}\right)$ over the pole. Thus,

$$
\begin{aligned}
\widehat{\mathcal{O}}^{(4)}= & \frac{4}{5}\left(\frac{2 n_{i}^{2}}{3}+1\right) \nabla_{i} \circ \mathrm{V}^{(4)^{\prime}}\left(z_{i}\right)+\left(-\frac{4 n_{i}^{3}}{15}-\frac{2 n_{i}}{5}\right) \sum_{j \neq i} \frac{n_{j}}{\left(z_{j}-z_{i}\right)^{2}} \mathrm{~V}^{(4)}\left(z_{i}\right) \\
& -\frac{4}{5} \nabla_{i}^{2} \circ \mathrm{V}^{(4)}\left(z_{i}\right)-\frac{n_{i}^{2}}{5}\left(\frac{1}{n_{i}}+\frac{2 n_{i}}{3}\right)^{2} \mathrm{~V}^{(4)^{\prime \prime}}\left(z_{i}\right)+\frac{n_{i}}{5} \sum_{j \neq i} \frac{n_{j}}{z_{j}-z_{i}} \mathrm{~V}^{(4)^{\prime}}\left(z_{i}\right) \\
& -\frac{1}{10} \partial_{i}^{2} \circ \mathrm{V}^{(2)}\left(z_{i}\right)+\frac{n_{i}^{2}}{5}\left(\frac{1}{n_{i}}+\frac{2 n_{i}}{3}\right)^{2} \sum_{r, s \geq 1}(r+1)(s+1) z_{i}^{-r-s-4} \frac{\partial^{2}}{\partial t_{r} \partial t_{s}} \\
& +\frac{n_{i}}{10}\left(\frac{1}{n_{i}}+\frac{2 n_{i}}{3}\right)^{2} \sum_{r \geq 1}(r+1)(r+2)(r+3) z_{i}^{-r-4} \frac{\partial}{\partial t_{r}} \\
& +\frac{1}{5}\left(\frac{1}{n_{i}}+\frac{2 n_{i}}{3}\right) \partial_{i} \circ \sum_{r \geq 1}(r+1)(r+2) z_{i}^{-r-3} \frac{\partial}{\partial t_{r}} \\
& -\frac{n_{i}}{5} \sum_{p \geq-1} z_{i}^{-p-2} \sum_{j \neq i} \frac{1}{z_{j}-z_{i}}\left(n_{i} \partial_{j}-n_{j} \partial_{i}\right) \circ \mathrm{L}_{p}^{(2)} .
\end{aligned}
$$

In the last term, there is clearly no pole, by virtue of (5.3).

Now, we replace the remaining $L^{(2)}$ with $L^{(4)}$, and add the difference to that part of (5.44) that is not factorized through the $\mathrm{L}^{(4)}$ yet. Rather surprisingly, this gives just

$$
\frac{n_{i}}{5}\left(\frac{2 n_{i}^{2}}{3}+1\right) \sum_{r \geq 1}(r+1) z_{i}^{-r-2} \sum_{p \geq-1} z_{i}^{-p-2} \frac{\partial}{\partial t_{r}} \circ \mathbf{L}_{p}^{(4)} .
$$

Thus all the terms in the decoupling operator have been factorized through $\mathrm{L}_{p}^{(4)}$ on the right: 


$$
\begin{aligned}
\widehat{\mathcal{O}}^{(4)}= & \frac{4}{5}\left(\frac{2 n_{i}^{2}}{3}+1\right) \nabla_{i} \circ \mathrm{V}^{(4)^{\prime}}\left(z_{i}\right)-\frac{2 n_{i}}{5}\left(\frac{2 n_{i}^{2}}{3}+1\right) \sum_{j \neq i} \frac{n_{j}}{\left(z_{j}-z_{i}\right)^{2}} \mathrm{~V}^{(4)}\left(z_{i}\right) \\
& -\frac{4}{5} \nabla_{i}^{2} \circ \mathrm{V}^{(4)}\left(z_{i}\right)-\frac{1}{5}\left(\frac{2 n_{i}^{2}}{3}+1\right)^{2} \mathrm{~V}^{(4)^{\prime \prime}}\left(z_{i}\right)-\frac{1}{10} \partial_{i}^{2} \circ \mathrm{V}^{(4)}\left(z_{i}\right) \\
& -\frac{n_{i}}{5} \sum_{p \geq-1} z_{i}^{-p-2} \sum_{j \neq i} \frac{1}{z_{j}-z_{i}}\left(n_{i} \partial_{j}-n_{j} \partial_{i}\right) \circ \mathrm{L}_{p}^{(4)} \\
& +\frac{n_{i}}{5}\left(\frac{2 n_{i}^{2}}{3}+1\right) \sum_{r \geq 1}(r+1) z_{i}^{-r-2} \sum_{p \geq-1} z_{i}^{-p-2} \frac{\partial}{\partial t_{r}} \circ \mathrm{L}_{p}^{(4)} .
\end{aligned}
$$

Along with eqs. (5.5) and (5.14), the background charge of the Virasoro generators $\mathrm{L}_{p}^{(4)}$ follows the general pattern (1.5) that was conjectured in [46].

Finally, since we are in fact considering $\left.\widehat{\mathcal{O}}^{(4)}\right|_{\text {inv }}$, the last formula can be rewritten as

$$
\widehat{\mathcal{O}}^{(4)}=\mathcal{A} \circ \sum_{p \geq-1} z_{i}^{-p-2} \mathrm{~L}_{p}^{(4)}
$$

with

$$
\begin{aligned}
\mathcal{A}= & \left(\frac{2 n_{i}}{5}-\frac{4 n_{i}^{3}}{15}\right) \sum_{j \neq i} \frac{n_{j}}{\left(z_{j}-z_{i}\right)^{2}}-\frac{4 n_{i}^{2}}{5} \sum_{j \neq i k \neq i} \sum_{\frac{n_{j} n_{k}}{\left(z_{j}-z_{i}\right)\left(z_{k}-z_{i}\right)}} \\
& +\left(\frac{4 n_{i}}{5}-\frac{8 n_{i}^{3}}{15}\right) \sum_{j \neq i} \frac{n_{j}}{z_{j}-z_{i}} \frac{\partial}{\partial z_{i}}-\frac{8 n_{i}^{2}}{5} \sum_{j \neq i} \frac{n_{j}}{z_{j}-z_{i}} \sum_{r \geq 1} z_{i}^{-r-1} \frac{\partial}{\partial t_{r}} \\
& -\frac{9 n_{i}^{2}}{10} \sum_{r, s \geq 1} z_{i}^{-r-s-2} \frac{\partial^{2}}{\partial t_{r} \partial t_{s}}-\frac{n_{i}^{2}}{5} \sum_{r \geq 1 s=1}^{r+1} \sum_{i}^{s-r-2} s t_{s} \frac{\partial}{\partial t_{r}} \\
& +\left(\frac{n_{i}^{3}}{3}-\frac{7 n_{i}}{10}\right) \sum_{r \geq 1}(r+1) z_{i}^{-r-2} \frac{\partial}{\partial t_{r}}+\left(n_{i}-\frac{8 n_{i}^{3}}{15}\right) \sum_{r \geq 1} z_{i}^{-r-1} \frac{\partial}{\partial t_{r}} \frac{\partial}{\partial z_{i}} \\
& +\left(-\frac{4 n_{i}^{4}}{45}+\frac{4 n_{i}^{2}}{15}-\frac{3}{10}\right) \frac{\partial^{2}}{\partial z_{i}^{2}},
\end{aligned}
$$

where $\partial / \partial z_{i}$ acts only on the explicit occurrences of $z_{i}$ in (5.47), while the $\partial / \partial t_{r}$ are composed with the $\mathrm{L}_{p}^{(4)}$.

Equations (5.46) constitute our result for the factorization of the '41' decoupling operator through the Virasoro generators. The pattern of the factorizations observed so far appears very 
convincing, and in Sect. 6 we suggest how the particular cases considered above fit into the general picture.

\subsubsection{The ' 22 ' decoupling operator}

A somewhat different situation occurs for the ' 22 ' case. One could expect a factorization of the decoupling operator through the Virasoro generators

$$
\mathrm{L}_{r \geq 1}^{(22)}=\frac{1}{2} \sum_{s=1}^{r-1} \frac{\partial^{2}}{\partial t_{s} \partial t_{r-s}}+\sum_{s \geq 1} s t_{s} \frac{\partial}{\partial t_{s+r}}-n_{i}(r+1) \frac{\partial}{\partial t_{r}}
$$

with the background charge taken from (4.26). However, applying the same strategy as above to the operator (4.27), we arrive at

$$
\begin{aligned}
& \widehat{\mathcal{O}}^{(22)}=\frac{1}{2} \nabla_{i} \circ \mathrm{V}^{(22)^{\prime}}\left(z_{i}\right)+\nabla_{i}^{2} \circ \mathrm{V}^{(22)}\left(z_{i}\right) \\
&+\left(n_{i}^{2}+2\right)\left\{-\frac{1}{2} \partial_{i}^{2} \circ \mathrm{V}^{(22)}\left(z_{i}\right)-n_{i} \sum_{j \neq i} \frac{1}{z_{j}-z_{i}}\left(n_{i} \partial_{j}-n_{j} \partial_{i}\right) \circ \mathrm{V}^{(22)}\left(z_{i}\right)-\frac{1}{4} \mathrm{~V}^{(22)^{\prime \prime}}\left(z_{i}\right)\right. \\
&\left(\frac{n_{i}^{3}}{2}+\frac{1}{2 n_{i}}\right) \sum_{j \neq i} \frac{n_{j}}{\left(z_{j}-z_{i}\right)^{2}} \mathrm{~V}^{(22)}\left(z_{i}\right)-\frac{n_{i}}{2} \sum_{r \geq 1}(r+1) z_{i}^{-r-2} \sum_{p \geq-1} z_{i}^{-p-2} \frac{\partial}{\partial t_{r}} \circ \mathrm{L}_{p}^{(22)} \\
&+\left.\frac{1}{4}\left(n_{i}-\frac{1}{n_{i}}\right)^{2} \sum_{j \neq i} \frac{n_{j}}{\left(z_{j}-z_{i}\right)^{2}} \sum_{r \geq 1}(r+1) z_{i}^{-r-2} \frac{\partial}{\partial t_{r}}\right\}
\end{aligned}
$$

and thus, although many cancellations (which have yet to be explained in an 'invariant' way have occurred en route from (4.27) to (5.49), the last term represents an obstruction to factorization. Its absence in the $(l, 1)$ case might be attributed to the special features enjoyed by the $(1, l)$ and $(l, 1)$ null vectors [25, 26, 27]. In Sect. 7 we comment briefly on why the $(l, 1)$ case is preferred by the Kontsevich-Miwa transform.

The obstruction would only vanish for a special value of $n_{i}$ (while in the $(l, 1)$ cases considered above, $n_{i}$ could be chosen as a free parameter). In view of eq. (4.24), this is $n_{i}=-1$, and therefore

16 Despite there being no complete factorization, the occurrence of the Virasoro generators with exactly the 'predicted' background charge (4.26) is quite remarkable. 


$$
Q=2, \quad d=-11
$$

Thus, finally, in this particular case we have the factorization

$$
\begin{aligned}
\widehat{\mathcal{O}}^{(22)}= & \frac{1}{2} \nabla_{i} \circ \mathrm{V}^{(\overline{22})^{\prime}}\left(z_{i}\right)+\nabla_{i}^{2} \circ \mathrm{V}^{(\overline{22})}\left(z_{i}\right) \\
& -\frac{3}{2} \partial_{i}^{2} \circ \mathrm{V}^{(\overline{22})}\left(z_{i}\right)+3 \sum_{j \neq i} \frac{1}{z_{j}-z_{i}}\left(\frac{3}{2} \partial_{j}-n_{j} \partial_{i}\right) \circ \mathrm{V}^{(\overline{22})}\left(z_{i}\right)-\frac{3}{4} \mathrm{~V}^{(\overline{22})^{\prime \prime}}\left(z_{i}\right) \\
& -3 \sum_{j \neq i} \frac{n_{j}}{\left(z_{j}-z_{i}\right)^{2}} \mathrm{~V}^{(\overline{22})}\left(z_{i}\right)+\frac{3}{2} \sum_{r \geq 1}(r+1) z_{i}^{-r-2} \sum_{p \geq-1} z_{i}^{-p-2} \frac{\partial}{\partial t_{r}} \circ \mathrm{L}_{p}^{(\overline{22})}
\end{aligned}
$$

with

$$
\mathbf{L}_{r \geq 1}^{(\overline{22})}=\frac{1}{2} \sum_{s=1}^{r-1} \frac{\partial^{2}}{\partial t_{s} \partial t_{r-s}}+\sum_{s \geq 1} s t_{s} \frac{\partial}{\partial t_{s+r}}+(r+1) \frac{\partial}{\partial t_{r}} .
$$

The numbers (5.50) are characteristic of the spin- $\frac{3}{2}$ ghosts 41. Thus, along with the two ghost systems participating in the diagram (1.8), another one is 'preferred' by the Kontsevich-Miwa transform.

Recall finally that the $d=-2$ matter corresponds to the set (4.28). Kontsevich-Miwa transforming the corresponding 'exceptional' decoupling operator does not require any additional calculations in view of the coincidences stated at the end of Sect. 4.2.2.

\section{Summary and generalizations}

The above can be summarized and generalized as follows. Let us start from the Virasoro constraints and ask ourselves whether they admit a transformation to the $z_{j}$ variables introduced according to (1.2). The answer depends on an interplay between the involved parameters, i.e. the 'spin' J entering the constraints, the Miwa parameter $n_{i}$, and an integer $l$, which is to become the level. For $l=2$ everything is very simple and the 'half' of the energy-momentum tensor itself, $\mathrm{T}_{\geq-1}\left(z_{i}\right)=\sum_{p \geq-1} z_{i}^{-p-2} \mathrm{~L}_{p}$, undergoes a transformation to the $z_{j}$ variables. However, for $l \geq 3$ the situation is different, as the Virasoro generators by themselves no longer allow a transformation into the $z_{j}$ variables, but only in higher-order combinations such as (5.16) or 
(5.46), however unnatural these may appear from the point of view of the $t$ variables. Fixing the coefficients appropriately and inverting the previous steps『, we arrive at the corresponding $(l, 1)$ decoupling equation in a dressed $\left(p^{\prime}, p\right)$ minimal model, where

$$
\frac{p^{\prime}}{p}=1+\frac{\mathrm{Q}^{2}}{4} \pm \frac{\mathrm{Q}}{4} \sqrt{\mathrm{Q}^{2}+8}, \quad \mathrm{Q} \equiv 2 \mathrm{~J}-1
$$

provided

$$
2 \mathrm{~J}-1 \equiv \mathrm{Q}=\frac{l-1}{\hat{n}}-\frac{2 \hat{n}}{l-1}, \quad(l \geq 2) .
$$

The integer $l$ specifies the $(l, 1)$ decoupling equation in the dressed minimal model. Equation (6.1) implies $(2 \mathrm{~J}-1)^{2}=2 \frac{\left(p-p^{\prime}\right)^{2}}{p p^{\prime}}$, which, however, is not a mere rewriting of the standard minimal model formula (3.4), since a priori $\mathrm{Q}=2 \mathrm{~J}-1$, as read off from the Virasoro constraints, need not coincide with the minimal model background charge $Q=\sqrt{\frac{1-d}{3}}$ (but it does as a result of the calculation).

To establish the correspondence starting from the minimal model's end, one first dresses the minimal model according to the 'Kontsevich-Miwa' dressing prescription which ensures that all operators have total dimensions proportional to their 'Liouville' charges (to be identified with the Miwa parameters):

$$
\Delta_{j}=-\frac{1}{2} Q n_{j}
$$

The matter dimensions are given by subtracting away the $U(1)$ Sugawara part, $\delta_{j}=\Delta_{j}-$ $\left(-\frac{1}{2} n_{j}^{2}\right)$, hence $n_{j}$ must be determined from the equation [which we have already met in (2.16)]

$$
n_{j}^{2}-Q n_{j}-2 \delta_{j}=0
$$

One also imposes the vanishing of the coefficient in front of the $I_{-1}^{l}$ term in the null vector build upon the $(l, 1)$ primary state. Then the order- $l$ decoupling equation corresponding to a given insertion of $\Psi \equiv \Psi_{l 1}$ at the point $z_{i}$,

\footnotetext{
${ }^{17}$ Technically, this can be done by evaluating contour integrals, as in 21.
} 


$$
\left\{\partial_{i}^{l}+\sum_{j \neq i} \frac{1}{z_{j}-z_{i}}\left(-l n_{i} n_{j} \partial_{i}^{l-1}+b_{j} \partial_{i}^{l-2} \partial_{j}+\ldots\right)+\ldots\right\}\left\langle\Psi\left(z_{i}\right) \prod_{j \neq i} \Psi_{j}\left(z_{j}\right)\right\rangle=0
$$

takes the form, after the Kontsevich-Miwa transform, of the constraint

$$
\left\{\sum_{n \geq-l+1} z_{i}^{-n-l} \mathbf{U}_{n}^{(l)}+\sum_{j \neq i} \frac{k_{j}}{z_{j}-z_{i}} \sum_{n \geq-l+2} z_{j}^{-n-l+1} \mathbf{U}_{n}^{(l-1)}+\ldots\right\} \tau(t)=0,
$$

where the omitted terms contain multiple poles, up to the last group of terms of order $(l-2)$, which enter multiplied with $\mathrm{U}_{n}^{(2)} \equiv \mathrm{L}_{n}$. All the $\mathrm{U}$ operators factorize through the $\mathrm{L}_{n}$ on the right. These Virasoro generators are of the form of (11.1) with $J$ fixed by the minimal model chosen, according to (6.1).

This suggests the correspondence between conformal field-theoretic ingredients and the KP tau function $\tau(t)$ to be achieved via the ansatz (1.4). More precisely, consider, in the dressed minimal model, correlators involving different primary fieldstళ,

$$
\tau(t) \equiv \tau\left\{z_{j}\right\}=\left\langle\prod_{a} \Psi_{l_{a} 1}\left(z_{i_{a}}\right)\right\rangle,
$$

where each of the $\Psi_{l_{a} 1}$ operators has an $\left(l_{a}, 1\right)$ null descendant, the 'matter' dimensions of the $\Psi_{l_{a} 1}$ being given by the RHS of (1.10). Then, eq. (6.4) (in which $Q=\sqrt{\frac{1-d}{3}}$ is fixed) for $j=i_{a}$ gives the respective 'Liouville' charges $n_{i_{a}}$ of the $\Psi_{l_{a} 1}$ :

$$
n_{i_{a}}^{2}=\left(l_{a}-1\right)^{2} \frac{13-d \pm \sqrt{(1-d)(25-d)}}{24}=\frac{\left(l_{a}-1\right)^{2}}{2}\left(\frac{p^{\prime}}{p}\right)^{ \pm 1}
$$

Now, the null states built over the $\left|\Psi_{l_{1}}\right\rangle$ give rise to the decoupling equations of the form of (6.5), the corresponding decoupling operator being $\partial_{i_{a}}^{l_{a}}+\ldots$. The possibility to KontsevichMiwa-transform each one of these decoupling equations depends only on the value of the respective parameter $n_{i_{a}}$. However, as we have just seen, the $n_{i_{a}}$ are such that each of them corresponds to the same background charge

\footnotetext{
${ }^{18}$ The appropriate insertions of the background charge and/or integrals of top forms of 'topological multiplets' are understood.
} 


$$
2 \mathrm{~J}-1=\frac{l_{a}-1}{n_{i_{a}}}-\frac{2 n_{i_{a}}}{l_{a}-1}=\sqrt{2} \frac{\left|p^{\prime}-p\right|}{\sqrt{p^{\prime} p}}
$$

and therefore all the corresponding decoupling operators factorize as

$$
\widehat{\mathcal{O}}_{i_{a}}^{\left(l_{a}\right)}=\mathcal{A}_{a} \circ \sum_{p \geq-1} z_{i_{a}}^{-p-2} \mathrm{~L}_{p}
$$

with the same Virasoro generators $\mathrm{L}_{p}$ for every $a$. Thus, a given set of Virasoro constraints implies all the $(l, 1)$ decoupling equations in the appropriate minimal model. Vice versa, it seems plausible that the full set of the $\widehat{\mathcal{O}}_{i_{a}}^{\left(l_{a}\right)}$-decoupling equations will imply the constraints $\sum_{p \geq-1} z_{i_{a}}^{-p-2} \mathrm{~L}_{p} \tau=0$ for every $a$, and these in turn would allow us to conclude that $\mathrm{L}_{p} \tau=0$, $p \geq-1[$ 四.

One may also view the dressing prescription (6.3) as a manifestation of the underlying BRST invariance in the realization of the topological algebra constructed in Sect. 3.1. The relation between the topological $U(1)$ charge of ghost-independent chiral primary states and the topological central charge,

$$
\mathrm{h}^{2}-(l-1) \frac{\mathrm{c}+3}{6} \mathrm{~h}+(l-1)^{2} \frac{\mathrm{c}-3}{6}=0
$$

gives

$$
n_{i}^{2}=\left\{\begin{array}{l}
(l-1)^{2} \frac{3-\mathrm{c}}{12} \\
(l-1)^{2} \frac{3}{3-\mathrm{c}}
\end{array}\right.
$$

Now, the equations (6.3) and (6.2) give, for $\Delta \equiv \Delta_{i}$, which is the matter+'Liouville' dimension of $\Psi$ :

$$
\Delta=\frac{n_{i}^{2}}{l-1}+\frac{1-l}{2}
$$

whence the 'minimal' dimension $\delta \equiv \delta_{i}$ is given by eq. (1.10).

\footnotetext{
${ }^{19}$ We are indebted to E. Kiritsis for a stimulating discussion of this point.
} 


\section{Concluding remarks}

Using the Kontsevich-Miwa transform, we have related the Virasoro constraints on the KP hierarchy to the highest-weight conditions (including the BRST invariance) in a realization of the topological (twisted $N=2$ ) algebra. In the representation, constructed in Sect. 3.1, of the topological algebra (1.11) in terms of matter, 'Liouville' and $c=-2$ ghost fields, the BRST invariance can be imposed level by level, by factoring away BRST-exact highest-weight vectors. In general, this gives an infinite set of equations on the correlators of various fields. The analysis carried out in this paper applies to the case when the correlators contain ghostindependent representatives of chiral primary fields (note the BRST operator (3.10) ) and the equations become the appropriately dressed $(l, 1)$ decoupling equations. These latter then factorize through the Virasoro generators that constrain the KP tau function. Unfortunately, we only observe this fact as an 'experimental evidence', and are unaware of its 'invariant' explanation.

It might be worth emphasizing that, although it is the level-2 decoupling operator (5.1) that essentially coincides with the Virasoro constraints, the higher-level decoupling operators do not factorize through the level-2 one (which would have been a contradiction); instead, when keeping $n_{i}=\hat{n}$ fixed, so as to have 'the same' Kontsevich-Miwa transform for every level, each of the $(l, 1)$ decoupling operators factorizes through a distinct, $l$-dependent, set of Virasoro generators $\mathrm{L}_{p \geq-1}^{(l)}$, whose background charge $\mathrm{Q}$ carries an explicit dependence on $l$ as given by (6.2). It is instructive, however, that technically, in order to prove the factorization, we were trying to factorize a given decoupling operator of a given level through the lower decoupling operators 'as far as possible'. The 'leftover', which accounts for the fact that the decoupling operator is not in the ideal generated by the lower ones, is such that, after the Kontsevich-Miwa transform, it serves precisely to 'correct' the background charges of the Virasoro generators involved.

To return to the interpretation in terms of differential operators, what we have observed can be stated as a theorem on 'meromorphic' differential operators of order $l$, of the form²

$$
\widehat{\mathcal{O}}=\partial_{i}^{l}+\sum_{j \neq i} \frac{1}{z_{j}-z_{i}}\left(a_{j} \partial_{i}^{l-1}+b_{j} \partial_{i}^{l-2} \partial_{j}+\ldots\right)+\ldots
$$

with vanishing zeroth-order part, in (infinitely many) variables $z_{j}$. Introducing parameters $t_{r}$

${ }^{20}$ To ensure that this corresponds to an $(l, 1)$ null state, one sets $a_{j}=-l n_{i} n_{j}$ with $n_{i}=(l-1) \sqrt{\frac{p^{\prime}}{2 p}}$. 
by eq. (1.2) and calling inv the subspace of those functions that depend on the $z_{j}$ only through the $t_{r}$, we have that the conditions

$$
\begin{aligned}
{\left[\widehat{\mathcal{O}}, \hat{\mathbf{L}}_{1}\right] } & \equiv \operatorname{0mod}\left(\hat{\mathbf{L}}_{\geq 1}, \hat{\mathbf{I}}_{\geq 1}\right), \\
{\left[\widehat{\mathcal{O}}, \hat{\mathbf{L}}_{2}\right] } & \equiv \operatorname{0mod}\left(\hat{\mathbf{L}}_{\geq 1}, \hat{\mathbf{I}}_{\geq 1}\right), \\
{\left[\widehat{\mathcal{O}}, \hat{\mathbf{I}}_{1}\right] } & \equiv \operatorname{0mod}\left(\hat{\mathbf{L}}_{\geq 1}, \hat{\mathbf{I}}_{\geq 1}\right),
\end{aligned}
$$

with

$$
\begin{aligned}
\hat{\mathbf{L}}_{1} & =\sum_{j}\left(z_{j}-z_{i}\right)^{2} \frac{\partial}{\partial z_{j}} \\
\hat{\mathbf{L}}_{2} & =\sum_{j}\left(z_{j}-z_{i}\right)^{3} \frac{\partial}{\partial z_{j}} \\
\hat{\mathbf{I}}_{1} & =\sum_{j} n_{j}\left(z_{j}-z_{i}\right)
\end{aligned}
$$

imply that $\left.\widehat{\mathcal{O}}\right|_{\text {inv }}$ factorizes through the Virasoro generators (1.1). This reformulation may provide an appropriate setting for proving the factorization in general.

An interesting possibility would be to study the implications for the conformal models of the Miwa-transformed [14, 15] Hirota bilinear relations. However, to prove that certain properties (e.g. fusion rules) of conformal models are equivalent (?) to Hirota-like identities, one has to take the latter in the version that, unlike the usual identities, involves Miwa parameters shifted as $n_{k} \mapsto n_{k} \pm n_{j}$ rather than $n_{k} \mapsto n_{k} \pm 1$. The demonstration of such 'discrete' identities would provide an independent and rigorous proof for our ansatz (6.7) for the tau function.

We have also seen that two different "bosonizations" of the type

$$
\text { twisted } N=2 \Longrightarrow \text { matter }+ \text { 'Liouville' + ghosts }
$$

are possible, resulting in different prescriptions to dress the matter. While the more standard DDK case is reproduced by taking spin-2 ghosts, it is the spin-1 ghost system that is required to prove the direct correspondence with the integrable formalism. Thus the topological algebra may be viewed as capturing the 'invariant' meaning of the theory, be it in the DDK formulation or in the guise of constrained integrable hierarchies.

Let us note that, according to the formula (1.9), the minimal-model values of the matter 
central charge $d$

$$
d=1-\frac{6\left(p^{\prime}-p\right)^{2}}{p^{\prime} p}
$$

come from the rational values

$$
\frac{\mathrm{c}}{3}=1-2\left(\frac{p}{p^{\prime}}\right)^{ \pm 1}
$$

of the topological central charge, so that for $p^{\prime}$ or $p=1$, the corresponding formula $\frac{c}{3}=1-\frac{2}{p}$ becomes that for the $N=2$ superconformal central charge $c=\frac{k}{k+2}, k=p-2$ (this point of view was elaborated recently in [53]). The thus distinguished rôle of the $(p, 1)$ models may explain the fact that only for the $(l, 1)$ decoupling operators there is no obstruction to the factorization through the Virasoro constraints.

Acknowledgements. We are grateful to L. Alvarez-Gaumé, B. A. Dubrovin, G. Felder, J. Fröhlich, A. Ganchev, K. Gawędzki, A. Gorsky, W. Lerche, A. Lossev, A. N. Schellekens, A. Turbiner and M. A. Vasiliev for useful discussions. We are specially indebted to R. Dijkgraaf, E. Kiritsis and A. A. Tseytlin for very useful suggestions. A. S. wishes to thank E. Corrigan for his kind hospitality at the I. Newton Institute, Cambridge. 


\section{References}

[1] E. Brézin and V. A. Kazakov, Phys. Lett. B236 (1990) 144.

[2] M. R. Douglas and S. H. Shenker, Nucl. Phys. B335 (1990) 635.

[3] D. J. Gross and A. A. Migdal, Phys. Rev. Lett. 64 (1990) 127.

[4] M. R. Douglas, Phys. Lett. B238 (1990) 176.

[5] M. Fukuma, H. Kawai and R. Nakayama, Int. J. Mod. Phys. A6 (1991) 1385; Comm. Math. Phys. 148 (1992) 106.

[6] R. Dijkgraaf, E. Verlinde and H. Verlinde, Nucl. Phys. B348 (1991) 435.

[7] M. Kontsevich, Funk. An. Prilozh. 25 (1991) No. 2, 50; Comm. Math. Phys. 147 (1992) 1.

[8] E. Witten, On the Konsevich Model and Other Models of Two-Dimensional Gravity, Princeton preprint IASSNS-HEP-91-24 (July 1991).

[9] E. Witten, Nucl. Phys. B340 (1990) 281.

[10] R. Dijkgraaf and E. Witten, Nucl. Phys. B342 (1991) 486.

[11] E. Verlinde and H. Verlinde, Nucl. Phys. B348 (1991) 457.

[12] A. M. Semikhatov, Virasoro Action and Virasoro Constraints on Integrable Hierarchies of the r-matrix Type, Lebedev Inst. preprint, hep-th/9112016; Nucl. Phys. B366 (1991) 347.

[13] T. Hollowood, J. Miramontes and J. Sánchez Guillén, Generalized Integrability and TwoDimensional Gravitation, CERN-TH.6678 (Oct. 1992).

[14] T. Miwa, Proc. Jpn. Acad. 58 (1982) 9.

[15] S. Saito, Phys. Rev. D 36 (1987) 1819; Phys. Rev. Lett. 59 (1987) 1798.

[16] E. Date, M. Jimbo, M. Kashiwara and T. Miwa, in: Proc. RIMS Symp. on Non-Linear Integrable Systems, M. Jimbo and T. Miwa (eds.) (World Scientific, Singapore, 1983), p. 39 .

[17] Yu. M. Makeenko and G. W. Semenoff, Properties of Hermitian Matrix Model in an External Field, British Columbia Univ. preprint PRINT-91-0329 (July 1991).

[18] S. Kharchev, A. Marshakov, A. Mironov, A. Morozov and A. Zabrodin, Nucl. Phys. B380 (1992) 181.

[19] D. J. Gross and M. J. Newman, Nucl. Phys. B380 (1992) 168.

[20] C. Itzykson and J.-B. Zuber, Combinatorics of the Modular Group II. The Kontsevich Integrals, Saclay preprint SPhT/92-001.

[21] A. M. Semikhatov, Nucl. Phys. B386 (1992) 139.

[22] V. G. Kač and A. S. Schwarz, Phys. Lett. B257 (1991) 329.

[23] B. L. Feigin and D. B. Fuchs, Funk. An. Prilozh. 16 (1982) No 2, 47.

[24] A. A. Belavin, A. M. Polyakov and A. B. Zamolodchikov, Nucl. Phys. B241 (1984) 333.

[25] L. Benoit and Y. Saint-Aubin, Phys. Lett. B215 (1988) 517.

[26] M. Bauer, Ph. Di Francesco, C. Itzykson and J.-B. Zuber, Nucl. Phys. B362 (1991) 515.

[27] A. Ch. Ganchev and V. B. Petkova, Phys. Lett. B293 (1992) 56.

[28] C. Gomez and G. Sierra, Phys. Lett. B255 (1991) 51. 
[29] A. Gorsky and K. Selivanov, Mod. Phys. Lett. A7 (1992) 2601.

[30] A. M. Polyakov, Conformal Turbulence, Princeton Univ. preprint PUPT-1341 (Sept. 1992).

[31] F. David, Mod. Phys. Lett. A3 (1988) 1651.

[32] J. Distler and H. Kawai, Nucl. Phys. B321 (1989) 509.

[33] S. Cecotti and C. Vafa, Ising Model and $N=2$ Supersymmetric Theories, HUTP-92/A044 (1992).

[34] B. A. Dubrovin, Geometry and Integrability of Topological-Antitopological Fusion, Comm. Math. Phys., to appear.

[35] E. S. Fradkin and A. A. Tseytlin, Phys. Lett. B158 (1985) 316; Nucl. Phys. B261 (1985) 1.

[36] R. Dijkgraaf, G. Moore and R. Plesser, The Partition Function of 2D String Theory, IASSNS-HEP-92/48 (Aug. 1992).

[37] W. Lerche, C. Vafa and N. P. Warner, Nucl. Phys. B324 (1989) 427.

[38] E. Witten, Commun. Math. Phys. 118 (1988) 411; Nucl. Phys. B340 (1990) 281.

[39] T. Eguchi and S.-K. Yang, Mod. Phys. Lett. A4 (1990) 1653.

[40] B. Gato-Rivera and A. M. Semikhatov, Phys. Lett. B293 (1992) 72.

[41] D. H. Friedan, E. J. Martinec and S. H. Shenker, Nucl. Phys. B271 (1986) 93.

[42] R. Dijkgraaf, H. Verlinde and E. Verlinde, Notes on Topological String Theory and 2d Quantum Gravity, PUPT-1217 (Nov. 1990).

[43] D. Friedan, Z. Qiu and S. Shenker, Phys. Rev. Lett. 52 (1984) 1575.

[44] Vl. S. Dotsenko and V. A. Fateev, Nucl. Phys. B240 (1984) 312.

[45] J. Distler, Nucl. Phys. B324 (1990) 523.

[46] B. Gato-Rivera and A. M. Semikhatov, Phys. Lett. B288 (1992) 38.

[47] A. M. Semikhatov, Int. J. Mod. Phys. A4 (1989) 467.

[48] P. G. Grinevich and A. Yu. Orlov, Flag spaces in KP Theory and Virasoro action on $\operatorname{det} \bar{\partial}_{j}$ and Segal-Wilson Tau Function, Cornell Univ. preprint CLNS 89/945.

[49] I. Krichever, Comm. Math. Phys. 143 (1992) 415.

[50] B. A. Dubrovin, Comm. Math. Phys. 145 (1992) 195.

[51] L. Brink, T. H. Hansson and M. A. Vasiliev, Phys. Lett. B286 (1992) 109.

[52] R. Dijkgraaf, private communication.

[53] M. Bershadsky, W. Lerche, D. Nemeschansky and N. P. Warner, Extended N = 2 Superconformal Structure of Gravity and W-Gravity Coupled to Matter, CERN-TH.6694/92.

[54] D. Gepner, Foundations of Rational Conformal Field Theory, I, CALT-68-1825 (Nov. 1992). 